\title{
Comparison of DES and URANS for Unsteady Vortical Flows over Delta Wings
}

\author{
L. A. Schiavetta* \\ K. J. Badcock ${ }^{\dagger}$ \\ University of Glasgow, Glasgow, G12 8QQ, UK University of Liverpool, Liverpool, L69 3GH, UK \\ R. M. Cummings $\ddagger$ \\ United States Air Force Academy, Colorado Springs, CO 80840 USA
}

\begin{abstract}
The unsteady behaviour of delta wing vortical flows is still a subject which is a challenge for numerical methods, such as computational fluid dynamics. New approaches to turbulence modelling, such as detached eddy simulation (DES) have been proposed which allow for greater accuracy of the numerical predictions. However, this increase in accuracy comes with a considerable increase in computational expense compared to traditional turbulence modelling. This investigation considers the use of both DES and unsteady Reynolds averaged Navier-Stokes (URANS) turbulence methods in the prediction of unsteady vortical flows. Calculations using DES are initially considered for two test cases and the predicted behaviour and resolution of the flow are considered. These results are then validated against existing experimental data, before the URANS calculations are considered with respect to the DES results. From this investigation it is determined that URANS turbulence models are able to predict the dominant features of the low frequency phenomenon present in the vortex system and in the post-breakdown region. Consideration is also given to the effect of grid refinement for both methods and the effect of temporal resolution for the DES calculations.
\end{abstract}

\section{Introduction}

$\mathrm{T}$ HE flow over delta wings is dominated by the leading edge vortices and other vortical structures. As the angle of incidence is increased the adverse pressure gradient within the flow causes vortex breakdown to occur over the wing. This flow can have a large impact on the behaviour of the wing and is found to be highly unsteady. For flight mechanics and aeroelastic behaviour, such as buffet, of existing configurations, it is clear that understanding the behaviour of unsteady forcing is crucial to allow the alleviation any structural response, which may exist. This is particularly important for complex fighter configurations such as the F16XL and EuroFighter and is compounded by the emergence of new UAV and UCAV technologies, which are tending toward planforms where unsteady vortical flows play a large role. This means that the need for a more complete understanding of the unsteady behaviour of vortical flows is becoming increasingly important.

To date, there has already been a great deal of research, which has considered the behaviour of this flow and what is generally known is summarised in the reviews by Gursul ${ }^{1}$ and by Nelson and Pelletier. ${ }^{2}$ From this research, it is clear that the unsteady behaviour of the vortical flow is complex, as a large number of flow phenomenon exist and interact, over and downstream of the wing. These flow phenomenon include the Helical mode instability of vortex breakdown, vortex wandering, vortex breakdown oscillations and shear layer instabilities. From consideration of the literature available, which considers the unsteady behaviour of the flow, patterns emerge relating the order and size of the non-dimensional frequencies with these flow features. Table 1 shows a summary of these features of the flow with their corresponding non-dimensional frequencies, taken from the literature.

*PhD Candidate, CFD Research Laboratory, Department of Aerospace Engineering, l.schiavetta@aero.gla.ac.uk

${ }^{\dagger}$ Professor of Computational Aerodynamics, FST Group, Department of Engineering

$\ddagger$ Professor of Aeronautics, Associate Fellow AIAA 
Table 1. Frequencies corresponding to important unsteady features of vortical flows

\begin{tabular}{lc}
\hline Phenomenon & Strouhal Number \\
\hline Helical Mode Instability & $1-2$ \\
Shear Layer Instabilities & $8-10$ and higher frequencies \\
Vortex Shedding - T.E & $\sim 8$ \\
Vortex Shedding - high $\alpha$ & $0.2-0.5$ \\
Vortex Breakdown Oscillation & $0.01-0.08$ \\
\hline
\end{tabular}

It was found that other dominant frequencies also featured in the literature, which were not clearly attributed to specific phenomenon. These are, $S t=2.5-4,5-6$ and the higher frequencies $\sim 20$. It is possible that these correspond to the phenomenon detailed above, however further investigation is needed. It is also important to note that there may be more than one dominant frequency associated with a particular phenonmenon, due to the complexity of the unsteady behaviour. For example, the shear layer instabilities will have at least two associated frequencies, this is due to the rolling up of the shear layer into discrete subvortices, which will have a frequency of rotation and also due to the movement of these structures around the vortex core. It may be difficult to separate these frequencies within a single solution, however, it may help to explain the spread of data and dominant frequencies assigned to particular flow features.

To allow for further understanding the behaviour of the flow, these phenomena can be split into two categories, those which occur upstream of breakdown and those occurring downstream. This is shown in Figure 1. Splitting the flow features in this way allows for an appreciation of which features will dominate, depending where vortex breakdown occurs on the wing. For breakdown close to the trailing edge, it is likely that the shear layer attachment and shear layer instabilities which would dominant the flow, however as the breakdown moves upstream, it is likely that the helical mode instability may dominant the flow frequency content. This will be important when considering the frequency content of the results and looking at the flow overall - particularly when considering the unsteady loading on the wing.

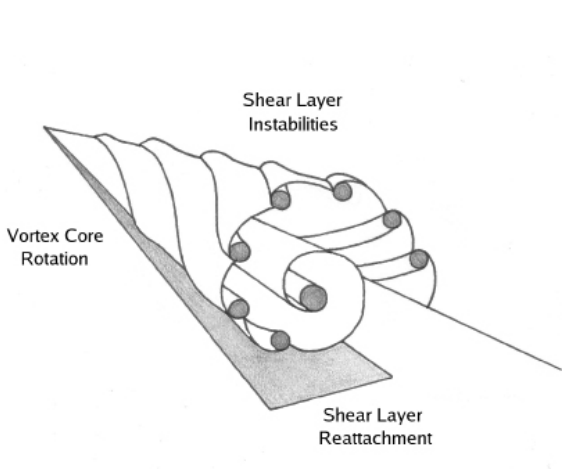

(a) Upstream

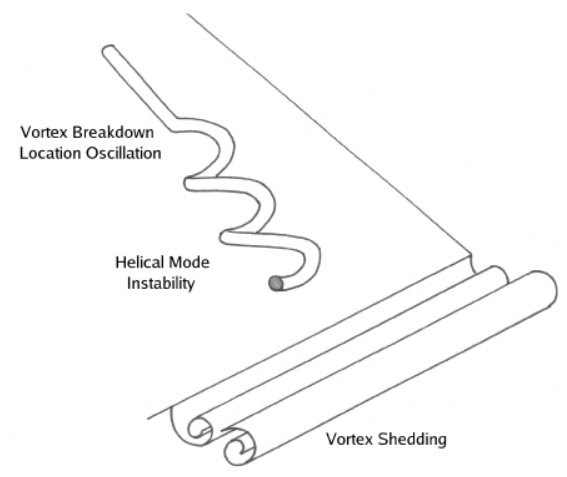

(b) Downstream

Figure 1. Schematic diagrams showing flow topology upstream and downsteam of vortex breakdown

Accurately predicting this complex flow is a challenge for numerical methods. In recent times the capabilities of CFD solvers have improved, with more complex turbulence modelling and treatments being utilised. One such method is Detached Eddy Simulation (DES), which is a hybrid URANS/LES turbulence treatment, proposed initially by Spalart ${ }^{3}$ to reduce the fine resolution of the grid in the boundary layer region needed for high Reynolds number LES calculations. It works by applying LES to the majority of the flow domain and URANS to the boundary layer region. This means that the majority of turbulence within the flow is simulated, with only the small scale eddies in the boundary layer being modelled. With this treatment many of the higher turbulent frequencies within the flow can be captured, which has led to a greater ability to predict more and more complex flow behaviour accurately. This has been shown in existing DES calculations on delta wing geometries, ${ }^{4-6}$ mostly carried out by the United States Air Force Academy (USAFA). 
However, DES is heavily dependent on both spatial and temporal refinement. Therefore, running a fully grid and time-step independent calculation is computationally expensive and for most CFD users, this is prohibitive.

From consideration of the literature, it is clear that the majority of the frequencies associated with the flow phenomena occur for Strouhal numbers less than 20. These lower frequencies are within the grasp of more traditional URANS calculations. Therefore, the question which this paper intends to address is whether DES is necessary to capture the lower frequencies of interest in the flow and if URANS can be used to capture the important frequencies and unsteady flow behaviours at a far reduced computational cost. To consider this problem, DES calculations were carried out by the University of Glasgow on a $70^{\circ}$ delta wing and the unsteady behaviour and flow resolution is considered and compared to a similar calculations performed at the USAFA for the same test case and for a $65^{\circ}$ wing. The results are then validated with experiment before the ability of URANS to predict the flow behaviour is analysed. The effect of the spatial and temporal refinement on the solutions is also considered before conclusions are made.

\section{Summary of Test Cases}

\section{A. $70^{\circ}$ ONERA Delta Wing}

The first test case used is a $70^{\circ}$ delta wing at an incidence of $\alpha=27^{\circ}$. Vortex breakdown occurs over the wing and there is an extensive database of experimental data, both time-averaged and unsteady for validation purposes. There is also a considerable database of computational results available for this configuration using both URANS and DES turbulence models ${ }^{4,5,7-9}$ from the NATO RTO Task group AVT-080 which considered "Vortex Breakdown over Slender Wings". ${ }^{10}$ The experimental data is taken from the $\mathrm{PhD}$ thesis by Mitchell ${ }^{11}$ and the associated papers. ${ }^{10,12-14}$ The experiments were carried out in two subsonic wind tunnels using a wide range of experimental techniques including, 3D Laser Doppler Anemometry (LDV), Particle Image Velocimetry (PIV) and data from both steady and unsteady pressure transducers $\left(\right.$ Kulites $\left.^{T M}\right)$.

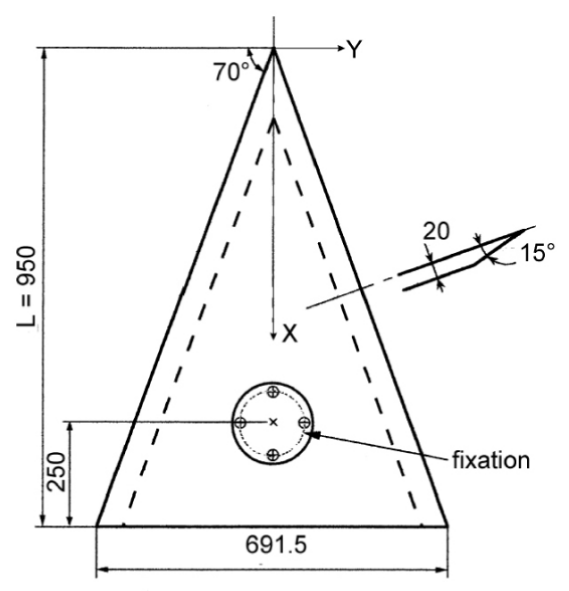

The wing used in the experiments has a root chord length of $950 \mathrm{~mm}$ and a sweep angle of $70^{\circ}$. It has flat upper and lower

Figure 2. $70^{\circ}$ ONERA Geometry (all distances marked are in $\mathbf{m m})^{11}$ surfaces with a $15^{\circ}$ bevel at the leading edge. The trailing edge is blunt with a thickness of $20 \mathrm{~mm}$. These details are shown in Figure 2. The experimental test conditions used by Mitchell were: an incidence of $\alpha=27^{\circ}$, a Mach number of $M=0.069$ and a Reynolds number based on the root chord of $R e=1.56 \times 10^{6}$. To help with the convergence of the compressible flow solver, the Mach number used for the investigation was raised to $M=0.2$. As this Mach number is still relatively low, this should not have a significant effect on the solution as compressibility effects will be negligible.

\section{B. $65^{\circ}$ VFE-2 Delta Wing}

The geometry used for the USAFA calculations is that currently being used for the 2nd International Flow Experiment (VFE-2) proposed by Hummel and Redecker, ${ }^{15}$ which is currently taking place as part of the NATO RTO AVT-113 Task Group. The geometry is originally from experiments carried out by Chu and Luckring $^{16}$ in the National Transonic Facility (NTF) at NASA Langley. These experiments considered a $65^{\circ}$ delta wing with four leading edge profiles (one sharp and three rounded with small, medium and large radii) for a wide range of conditions both subsonic and transonic and for both test and flight Reynolds numbers. This data has been compiled into a comprehensive experimental database and forms the basis for the investigations of the VFE-2. The geometry is analytically defined for all leading edge profiles, 
which allows improved correlation between experimental and computational results by reducing geometrical discrepancies. For this investigation, only the sharp leading edge profile is considered. Figure 3 shows the wing situated in the NTF wind tunnel and a brief overview of the analytical dimensions of the wing. All calculations were performed at a Mach number of $M=0.85$ and Reynolds number, based on the mean aerodynamic chord, of $R e=6 \times 10^{6}$ and for an incidence of $\alpha=23^{\circ}$.

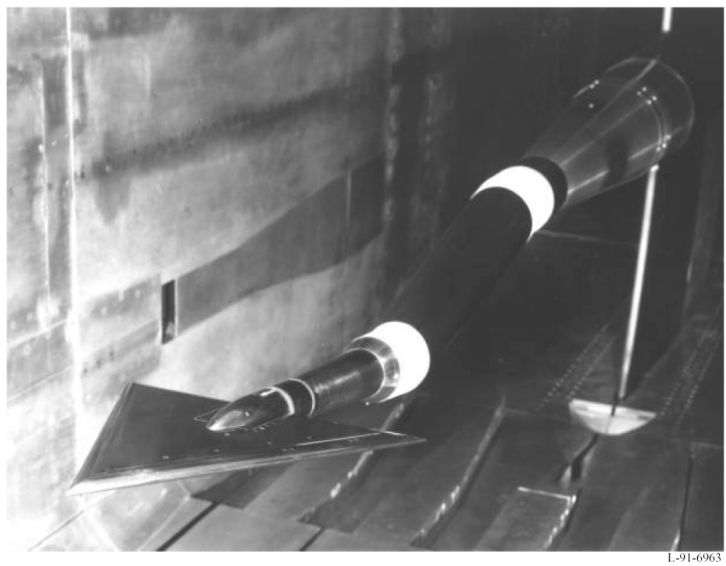

(a) Wing in NASA's NTF tunnel

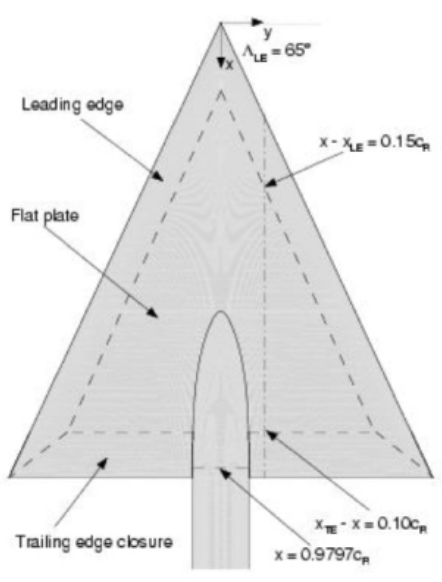

(b) Analytical definition of sharp leading edge case

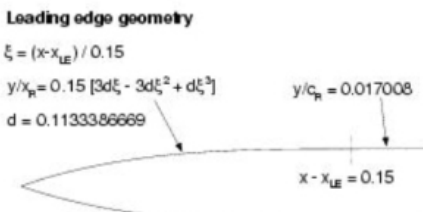

Trailng edge geometry

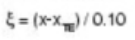

$y / c_{R}=0.017008 \quad y / x_{R}=-0.10\left[3 d \xi-3 d \xi^{2}+d \xi^{3}\right\}$

$d=0.1700800036901$

$x-x_{L E}=0$

Figure 3. Wing geometry used in investigation ${ }^{16}$

\section{Computational Methods}

\section{A. Flow Solver}

\section{University of Glasgow}

The PMB (Parallel Multi-Block) code is a multi-block structured solver which solves the unsteady RANS equations in a 3D Cartesian frame of reference. ${ }^{17}$ The governing equations are discretized using a cellcentred finite volume approach combined with an implicit dual-time method. In this manner, the solution marches in pseudo-time for each real time-step to achieve fast convergence. Two methods are available for the discretisation of the convective terms, either Osher's upwind scheme ${ }^{18}$ or Roe's flux-splitting scheme. ${ }^{19}$ MUSCL interpolation is used to provide nominally third order accuracy and the van Albada limiter is also applied to remove any spurious oscillations across shock waves. Central differencing is used to discretise the viscous terms, with the resulting non-linear system of equations generated being solved by integration in pseudo-time using a first-order backward difference. A Generalised Conjugate Gradient method is then used in conjunction with a Block Incomplete Lower-Upper (BILU) factorisation as a pre-conditioner to solve the linear system of equations, which is obtained from a linearisation in pseudo-time. A number of one and two equation turbulence models are available in the solver, as well as the option of Large Eddy Simulation (LES) and Detached Eddy Simulation (DES). However, in this investigation two models are used, the $k-\omega$ with $P_{\omega}$ enhancer model which incorporates a rotation correction into the Wilcox $k-\omega$ model and SA-DES which uses the Spalart-Almaras model as the URANS part of DES.

\section{USAFA}

Solutions for all configurations were computed with the commercial version of Cobalt developed by Cobalt Solutions, LLC. Cobalt solves the unsteady, three-dimensional, compressible Navier-Stokes equations on a hybrid unstructured grid. Full details of the computational scheme are presented in Ref. 20. The code has several choices of turbulence models, which include a number of URANS models, as well as many versions of DES using various URANS models. All simulations were computed on unstructured meshes with prisms in the boundary layer and tetrahedra elsewhere. In this investigation, the SA-DES model is used. Details of all the models used in this investigation are given in the following section. 


\section{B. Turbulence Models}

\section{1. $k-\omega$ with $P_{\omega}$ Enhancer Model}

The $k-\omega$ model is a two equation Boussinesq based turbulence model. ${ }^{21}$ This model uses the flow parameters, $k$, specific turbulent kinetic energy and, $\omega$, the specific dissipation rate per unit turbulent kinetic energy to calculate the eddy viscosity and to close the Reynolds averaged Navier-Stokes equations. The kinematic eddy viscosity for this model is given by,

$$
\mu_{T}=\rho \frac{k}{\omega}
$$

To calculate the turbulent kinetic energy and dissipation rate, two transport equations are added to the Navier-Stokes equations. The transport equations take the form,

$$
\rho \frac{\partial k}{\partial t}+\frac{\partial \rho k U_{j}}{\partial x_{j}}=\frac{\partial}{\partial x_{j}}\left[\left(\mu+\sigma^{*} \mu_{T}\right) \frac{\partial k}{\partial x_{j}}\right]+\rho P_{k}-\beta^{*} \rho k \omega
$$

and,

$$
\rho \frac{\partial \omega}{\partial t}+\frac{\partial \rho \omega U_{j}}{\partial x_{j}}=\frac{\partial}{\partial x_{j}}\left[\left(\mu+\sigma \mu_{T}\right) \frac{\partial \omega}{\partial x_{j}}\right]+\rho P_{\omega}-\beta \rho \omega^{2}
$$

where the production terms are given by

$$
P_{k}=\tau_{i j} \frac{\partial U_{i}}{\partial x_{j}} \quad P_{\omega}=\alpha^{*} \frac{\omega}{k} P_{k}
$$

The model coefficients are given in Table 2 .

Table 2. Model constants for the $k-\omega$ turbulence model ${ }^{21}$

\begin{tabular}{ccccc}
\hline$\alpha^{*}$ & $\beta$ & $\beta^{*}$ & $\sigma$ & $\sigma^{*}$ \\
$5 / 9$ & 0.075 & 0.09 & 0.5 & 0.5 \\
\hline
\end{tabular}

It is well known that the standard model, as with most other Boussinesq models, over-predicts the eddy viscosity within the vortex core which leads to exaggerated diffusion of vorticity. This is due to the fact that only the strain rate is considered in the calculation of $\tau_{i j}$ using the Boussinesq approximation, however vortical flows are highly rotational. The enhanced model which was proposed by Brandsma et al. ${ }^{22}$ controls the production of turbulent kinetic energy and hence eddy viscosity through an increase in the production of the dissipation rate $(\omega)$ within the regions of highly rotational flow. A suitable sensor has been used to distinguish between shear layers and vortex cores. This sensor considers the ratio of the magnitude of the strain rate tensor to the magnitude of the rotation tensor defined below as,

$$
r=\frac{S^{*}}{\Omega}=\frac{\left(2 S_{i j}^{*} S_{i j}^{*}\right)^{1 / 2}}{\left(2 \Omega_{i j} \Omega_{i j}\right)^{1 / 2}}
$$

where

$$
S_{i j}^{*}=\frac{1}{2}\left(\frac{\partial U_{i}}{\partial x_{j}}+\frac{\partial U_{j}}{\partial x_{i}}\right)-\frac{1}{3} \frac{\partial U_{k}}{\partial x_{k}} \delta_{i j} \quad \text { and } \quad \Omega_{i j}=\frac{1}{2}\left(\frac{\partial U_{i}}{\partial x_{j}}-\frac{\partial U_{j}}{\partial x_{i}}\right)
$$

In shear layers, the velocity gradient is dominated by the normal gradients such that $r \approx 1$, whereas in the core of the vortex the flow approaches a purely rotational state, which implies that $r<<1$. Thus, this correction for vortical flows uses this relationship to determine the vortical regions and enhances the production of the dissipation in order to reduce the turbulence in vortex cores. This is achieved by dividing the production of $\omega$ by $\min \left(r^{2}, 1\right)$ as,

$$
P_{\omega_{\text {new }}}=\frac{P_{\omega}}{\min \left(r^{2}, 1\right)}
$$

Investigations have been carried out using this model for both subsonic ${ }^{7}$ and transonic ${ }^{22,23}$ conditions. 


\section{Spalart-Allmaras Model}

The Spalart-Allmaras ${ }^{24}$ one equation model (SA) solves a single partial differential equation for a working variable $\tilde{\nu}$ which is related to the turbulent viscosity. The differential equation is derived by "using empiricism and arguments of dimensional analysis, Galilean invariance and selected dependence on the molecular viscosity." ${ }^{24}$ The model includes a wall destruction term that reduces the turbulent viscosity in the laminar sublayer. The model takes the form,

$$
\frac{\partial \tilde{\nu}}{\partial t}+\frac{\partial\left(\tilde{\nu} u_{j}\right)}{\partial x_{j}}=c_{b 1} \tilde{S} \tilde{\nu}-c_{w 1} f_{w}\left(\frac{\tilde{\nu}}{d}\right)^{2}+\frac{1}{\sigma} \frac{\partial}{\partial x_{k}}\left[(\nu+\tilde{\nu}) \frac{\partial \tilde{\nu}}{\partial x_{k}}\right]+\frac{c_{b 2}}{\sigma} \frac{\partial \tilde{\nu}}{\partial x_{k}} \frac{\partial \tilde{\nu}}{\partial x_{k}}
$$

The turbulent kinematic viscosity is obtained from,

$$
\nu_{T}=\frac{\mu_{T}}{\rho}=\tilde{\nu} f_{v 1}, \quad \text { where } \quad f_{v 1}=\frac{\chi^{3}}{\chi^{3}+c_{v 1}^{3}} \quad \text { and } \quad \chi=\frac{\tilde{\nu}}{\nu}
$$

where $\mathrm{S}$ is the magnitude of the vorticity given by

$$
S=|\omega|=|\nabla \times(u \hat{i}+v \hat{j}+w \hat{k})|
$$

and the modified vorticity is,

$$
\tilde{S}=S+\frac{\tilde{\nu}}{\kappa^{2} d^{2}} f_{v 2} ; \quad f_{v 2}=1-\frac{\chi}{1+\chi f_{v 1}} ;
$$

where $\mathrm{d}$ is the distance to the closest wall. The wall destruction function $f_{w}$ is,

$$
f_{w}=g\left[\frac{1+c_{w 3}^{6}}{g^{6}+c_{w 3}^{6}}\right]^{1 / 6}, \quad \text { with } \quad g=r+c_{w 2}\left(r^{6}-r\right) \quad \text { and } \quad r=\frac{\tilde{\nu}}{\tilde{S} \kappa^{2} d^{2}}
$$

The turbulent viscosity is obtained from the turbulent kinematic viscosity by $\mu_{T}=\rho \nu_{T}$. The model coefficients are given in Table 3.

Table 3. Model coefficients for the Spallart-Allmaras turbulence model, ${ }^{\mathbf{2 4}}$ where $c_{\mathrm{w} 1}=\frac{c_{\mathrm{b} 1}}{\kappa^{2}}+\frac{\left(1+c_{\mathrm{b} 2}\right)}{\sigma}$

\begin{tabular}{cccccccccccc}
\hline$c_{b 1}$ & $c_{b 2}$ & $c_{v 1}$ & $c_{w 1}$ & $c_{w 2}$ & $c_{w 3}$ & $c_{t 1}$ & $c_{t 2}$ & $c_{t 3}$ & $c_{t 4}$ & $\sigma$ & $\kappa$ \\
0.1355 & 0.622 & 7.1 & 3.239 & 0.3 & 2.0 & 1.0 & 2.0 & 1.1 & 2.0 & $2 / 3$ & 0.41 \\
\hline
\end{tabular}

\section{Detached-Eddy Simulation}

The Detached-Eddy Simulation method was proposed by Spalart et al. ${ }^{3}$ and was originally based on the Spalart-Allmaras one equation RANS turbulence model (detailed above) with a more detailed presentation in Ref. 24. The wall destruction term presented above is proportional to $(\tilde{\nu} / d)^{2}$, where $d$ is the distance to the wall. When this term is balanced with the production term, the eddy viscosity becomes proportional to $\hat{S} d^{2}$, where $\hat{S}$ is the local strain rate. The Smagorinsky LES model varies its sub-grid scale (SGS) turbulent viscosity with the local strain rate, and the grid spacing: $\nu_{S G S} \propto \hat{S} \Delta^{2}$, where $\Delta=\max (\Delta x, \Delta y, \Delta z)$. If $d$ is replaced with $\Delta$ in the wall destruction term, the Spalart-Almaras model will act as a Smagorinsky LES model. To exhibit both URANS and LES behavior, $d$ in the Spalart-Almaras model is replaced by

$$
\tilde{d}=\min \left(d, c_{D E S} \Delta\right)
$$

When $d>>\Delta$, the model acts in a RANS mode and when $d>>\Delta$ the model acts in a Smagorinsky LES mode. Therefore, the model switches into LES mode when the grid is locally refined. DES was implemented in an unstructured grid method by Forsythe et al. ${ }^{25}$ where it was determined that the DES constant should be $c_{D E S}=0.65$, consistent with the structured grid implementation of Spalart et al. ${ }^{3}$ when the grid spacing, $\Delta$, was taken to be the longest distance between the cell center and all of the neighboring cell centers. 


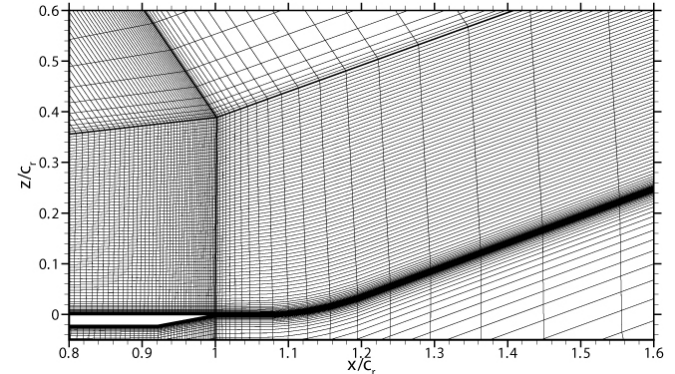

(a) Fine grid

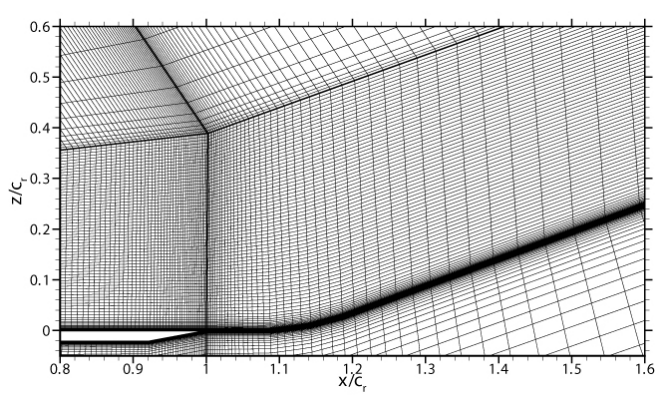

(b) Fine grid with refined trailing edge

Figure 4. Comparison of grid refinement in trailing edge region for fine grid and refined TE grids

\section{Grid Generation}

\section{University of Glasgow}

The structured multi-block grid of the University of Glasgow was created using the Icemcfd mesh generation package, Hexa. The wing geometry was altered to include a $15^{\circ}$ bevel, similar to the leading edges. A semi-span H-H grid toplogy with no sting arrangement was used, which sets the incidence of the wing to $27^{\circ}$ in the grid. The grid also uses a "collapsed apex" blocking strategy, where the edges of the blocks in the wing apex region have been collapsed to create a singular point. This grid topology has been used successfully in a number of investigations using RANS turbulence models by Allan. ${ }^{7}$ The far field was defined $20 c_{r}$ in each direction from the wing apex to minimise the effect of the boundaries on the flow. Convergence problems associated with the singularity were dealt with by using laminar flow at the apex and fixing transition to turbulence at a constant streamwise location in the grid. This location was was set to $x=0.4$ for all calculations based on the investigation carried out by Morton ${ }^{5}$ for the same test case. This value of transition corresponds to a value of $x / c_{r}=0.35914$ on the wing upper surface.

Three grids are used in the calculations by the University of Glasgow. Using one of the grids created by Allan as a starting point, the initial two grids were created with differing levels of refinement, coarse and fine, with $3.97 \times 10^{6}$ and $7.77 \times 10^{6}$ grid points respectively. Both grids have a first wall spacing of $1 \times 10^{-6} c_{r}$, which corresponds to a $y^{+}$value of approximately 0.1 and a stretching ratio, within the boundary layer region, of 1.2. The $y^{+}$value is sufficient for the flow conditions and the stretching ratio is within the recommended range for adequate log-layer resolution suggested for RANS calculations by Spalart. ${ }^{26}$ The fine grid has a higher concentration of points in these regions than the coarse grid and also has a much improved orthogonality over the whole area of interest close to the wing. The third grid was created to consider the effect of refinement in the trailing edge region on the upstream vortical flow for the DES solutions. This grid was based on the fine grid with the same distribution of points over the wing. However,

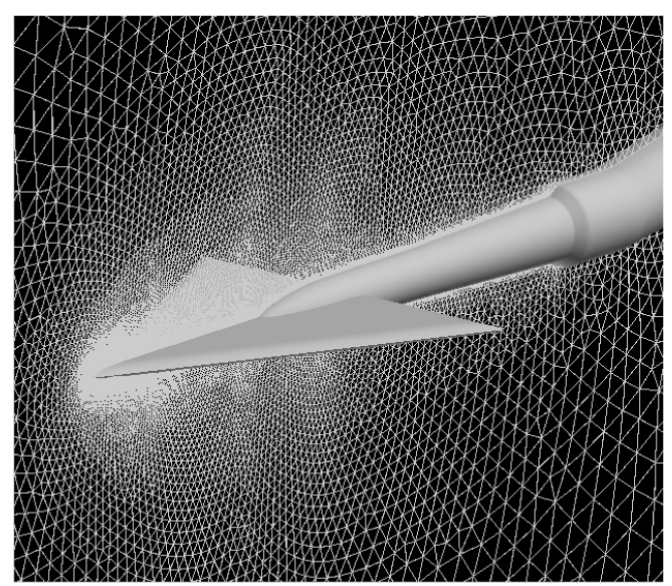

Figure 5. USAFA grid at symmetry plane in the region downstream of the trailing edge more grid points were added and the stretching ratio was decreased in the streamwise direction to improve the refinement. The resulting grid had $8.77 \times 10^{6}$ grid points. Figure 4 shows the grid refinement in this region for the two grids. 


\section{USAFA}

The computational meshes at USAFA were generated with the software packages GridTool ${ }^{27}$ and VGRIDns ${ }^{28}$ developed at NASA Langley Research Center. For the $65^{\circ}$ VFE-2 test case, the calculations were carried out on an unstructured grid which had approximately $7.89 \times 10^{6}$ cells and an average first wall spacing of $y^{+}=0.68$, created specifically for a Reynolds number of $6 \times 10^{6}$. The grid was refined within the vortex core region to improve the grid for the application of DES. The grid structure at the symmetry plane is shown in Figure 5.

\section{Assessment of DES Results}

The DES results from the University of Glasgow, used for the majority of this investigation, were obtained using the fine grid with a time step of $\Delta \tau=0.0025$. This will be considered along with the DES results from the USAFA calculations for the $65^{\circ}$ VFE-2 test case, which were carried out using a non-dimensional time step of $\Delta \tau \approx 0.0047$. Further analysis was performed on these results to consider the unsteady behaviour of the flow and the ability of DES to predict this behaviour. The sensitivity of these results to grid and time step refinement will be considered in a later section.

To consider the unsteady behaviour of the results a number of point probes were situated in the computational domain. These were placed along a constant conical ray from the apex of the wing in each case, close to the vortex core axis. For the ONERA test case five probes were used, placed at streamwise locations $x / c_{r}=0.53,0.63,0.74,0.84$ and 1.00 at a position, $z / c_{r}=0.1$, above the wing surface. Two probes were used for the VFE-2 test case at streamwise locations of $x / c_{r}=0.7$ and 0.9 , again $z / c_{r}=0.1$ above the wing surface. The locations of these probes in relation to the flow features for both cases are shown in Figure 6.

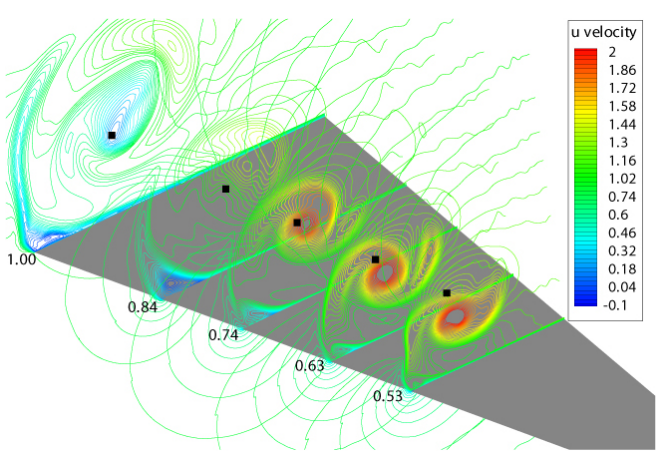

(a) $70^{\circ}$ ONERA test case with slices showing contours of $u$ velocity

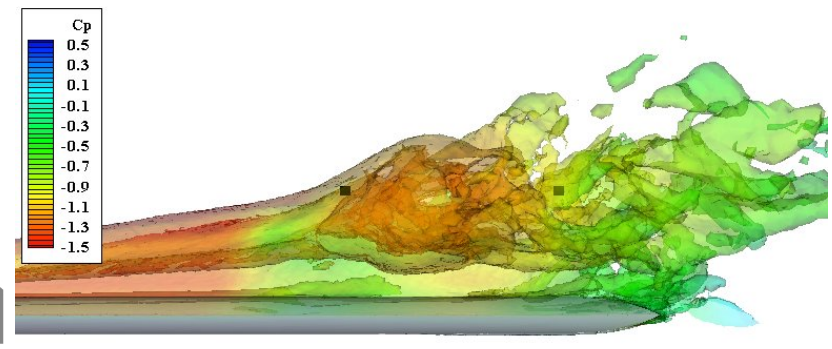

(b) $65^{\circ} \mathrm{VFE}-2$ test case with an isosurface of $x$ vorticity coloured by $\mathrm{Cp}$

Figure 6. Location of probes though vortex core region with reference to flow behaviour for both test cases

For the Glasgow results using the $70^{\circ}$ ONERA test case, the mean location of vortex breakdown was found to occur at approximately $x / c_{r}=0.86$. Therefore, the probes at $x / c_{r}=0.53,0.63$ and 0.74 are upstream of vortex breakdown, with the probe at $x / c_{r}=0.53$ sitting above the vortex core within the shear layer, and the probes at $x / c_{r}=0.63$ and 0.74 located close to the vortex core. The probe at $x / c_{r}=0.84$ also sits within the vortex core and is close to the vortex breakdown location and the probe at $x / c_{r}=1.0$ is downstream of breakdown, below the vortex core winding.

For the USAFA results using the $65^{\circ}$ VFE-2 test case, the mean location of vortex breakdown occurs at $x / c_{r}=0.68$. This means that the probe at $x / c_{r}=0.7$ is close to breakdown and $x / c_{r}=0.9$ is in the post-breakdown region. Although the locations of these probes are quite different from the ONERA test case, the non-dimensional distance from the vortex breakdown are similar to the $x / c_{r}=0.84$ and 1.0 probes. Therefore, a qualitative comparison of the behaviour may be made for these locations. Keeping all these locations in mind, each of the velocity components were analysed, to determine the behaviour of the flow upstream, at, and downsteam of breakdown. However, only the $u$ velocity component results will be shown here. 


\section{A. Unsteady Behaviour of DES Solution}

We first consider the 70 ONERA test case. The results of the unsteady analyses are shown in Figure 7. At $x / c_{r}=0.53$, the time history exhibits a reltively large amplitude periodic oscillation with a high frequency. Closer to the vortex core at $x / c_{r}=0.63$ and 0.74 , the signal oscillation becomes more irregular and the amplitude decreases significantly. This reduction in amplitude is consistent with the reduction of the RMS values given in Figure $7(\mathrm{a})$ for these locations. At $x / c_{r}=0.84$, the time history changes significantly from the upstream probes, with a high amplitude, low frequency oscillation being dominant. This also coincides with a large increase in RMS velocity, however the mean velocity has decreased. The mean velocity is positive for this location, however, from the time history it is evident that the flow does reverse and therefore breakdown crosses the probe location. Downstream of the breakdown location the amplitude decreases and a more periodic waveform returns. The mean velocity at this point has only increased slightly compared to the $x / c_{r}=$ 0.84 location, however the RMS value has decreased and there is no recirculation in this region (the $u$ velocity does not become negative).

Considering the frequency content of the signals given by the PSD plots, a number of dominant frequencies at each of the probe locations are clear. The most dominant frequency found in the flow occurs for the probe at $x / c_{r}=0.84$ at a non-dimensional frequency of approximately $S t=f c_{r} / U_{\infty}=0.07$. Two other low frequencies are also apparent at $S t \approx 0.27$ and 0.67 . There is little energy at higher frequencies at this location. As vortex breakdown has been shown to oscillate across this probe location, it is likely that this low frequency is caused by the oscillation of the vortex

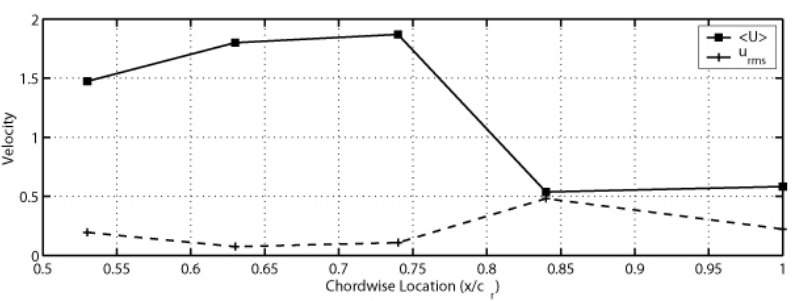

(a) Mean and RMS behaviour

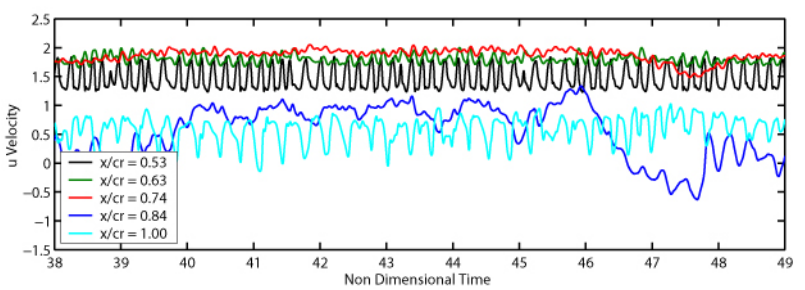

(b) Time history

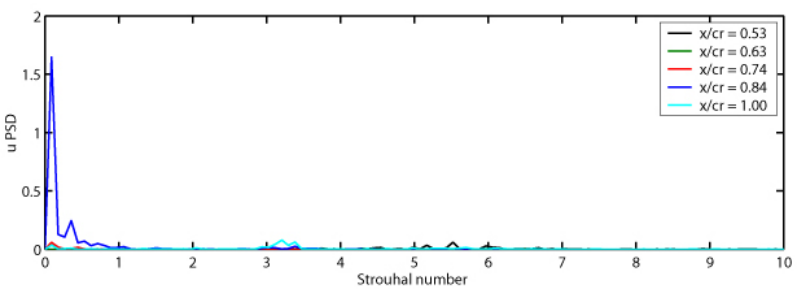

(c) PSD analysis

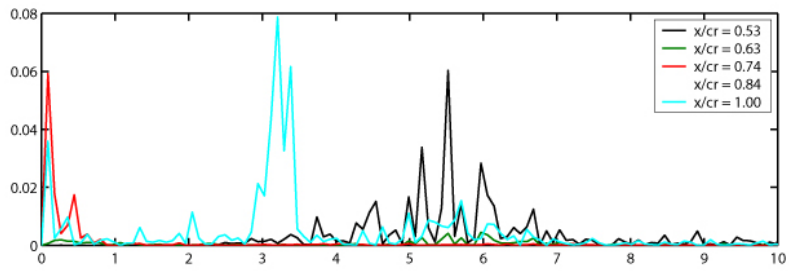

(d) PSD analysis with $x / c_{r}=0.84$ probe signal removed for clarity of frequency content of remaining probes

Figure 7. Unsteady behaviour of non-dimensional $u$ velocity components at probes through vortex core region shown by Mean, RMS, time histories and PSD frequency plots for $70^{\circ}$ ONERA test case

breakdown location in the flow. As the energy in this low frequency is large, relative to the other probe signals, Figure $7(\mathrm{~d})$ shows the same PSD plot with the $x / c_{r}=0.84$ signal removed for clarity. It is clear that at $x / c_{r}=0.53$, the high frequency content corresponds to frequencies in the range $S t=4.5-6$, with energy content also present at $S t \approx 9$. It is suggested that these frequencies are due to shear layer instabilities, such as the Kelvin-Helmholtz instability. Downstream, close to the vortex core, the energy in this frequency range is lower. Finally, downstream of breakdown, a new range of dominant frequencies are found. These occur in the range $S t=3-3.5$ and can be associated with the helical mode instability.

From this analysis, it is clear that there are a number of identifiable features in the flow, both upstream and downstream of the breakdown location with relatively low frequencies. Upstream, the flow is dominanted by a strong vortical system, containing both primary and secondary vortices. Close to the vortex core this flow exhibits only small fluctuations. Within the shear layer, possible evidence of shear layer roll 
up instabilities, such as the Kelvin-Helmholtz type instability have been found from the frequency data at $S t=4.5-6$. At breakdown, the flow is dominated by the motion of the breakdown location which oscillates in the streamwise and spanwise direction at a very low non-dimensional frequency at $S t=0.07$. Both these phenomenon have been found to occur for a range of configurations and do not appear to be dependent on turbulence.

Downstream of breakdown, the helical mode instability is present and the frequencies corresponding to its rotation and general behaviour have been isolated in the range $S t=3-3.5$. It is in the post-breakdown flow where turbulent behaviour is expected to be found as the vortex breaks down and loses its structure. However, from these results it is clear that the helical mode winding exhibits coherent periodic behaviour, and therefore is not driven turbulent phenomenon. Further evidence of this may be obtained from the results of a highly under-resolved (both spatially and temporally) DES calculation which was performed on the coarse grid using a time step of $\Delta \tau=0.01$. Using such a coarse calculation, it is not likely that any small scale fluctuations will be captured and indeed from the time history and PSD plot for the same probe location shown in Figure 8 it is clear that none are found. The PSD analysis shows that the frequency of the helical mode is identical for this case and although no small scale structures were
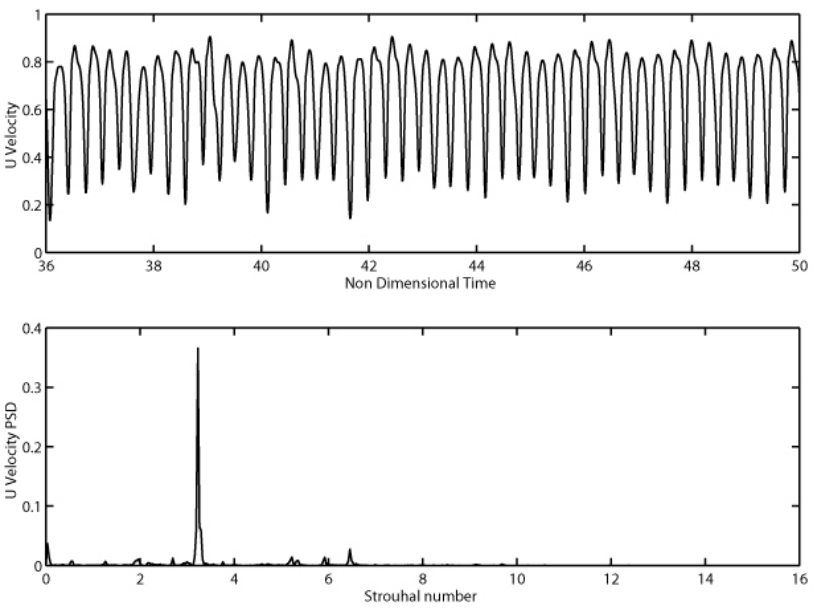

Figure 8. Non-dimensional $u$ velocity time history and PSD for a probe on the vortex axis, downstream of vortex breakdown, from a highly under-resolved DES solution, Coarse Grid, $\Delta \tau=0.01$ for $70^{\circ}$ ONERA test case captured there was little effect on the prediction of the vortex breakdown winding and its frequency.

This conclusion is also confirmed from consideration of the literature. A number of numerical investigations have been performed using both inviscid ${ }^{9}$ and laminar ${ }^{29}$ methods, which clearly show the helical mode instability behaviour.

Now we consider the $65^{\circ}$ VFE-2 test case. Figure 9 shows the unsteady analysis results. These results are compared to the probes at $x / c_{r}=0.84$ and 1.00 for the $70^{\circ}$ results detailed before and shown in the same colours in Figure 7. From the time histories, it is clear that there are many similarities with the $70^{\circ}$ results. In both cases, the location of vortex breakdown periodically moves upstream of the pre-breakdown probe location. This is evident from the low frequency, high amplitude behaviour and the magnitude of the traces periodically reducing to less than zero, indicating reversed flow. The fluctuations of the location of breakdown seem to be more pronounced for the $65^{\circ}$ test case, however this is likely to be due to the presence of shocks in the flow, which have been found to move abruptly. ${ }^{30,31}$ It is also clear that when the breakdown location is upstream of the probe, there is less unsteadiness in the flow. Considering the frequency content of the solutions at the upstream probe, it is clear that the dominant frequency of the flow close to breakdown is approximately $S t=0.1$, which is in good agreement with the $70^{\circ}$ test case for the frequency associated with the movement of breakdown. However, the power of the signal for the $65^{\circ} \mathrm{VFE}-2$ test case at this frequency is greater, again likely due to the occurrence of shocks in the flow.

Downstream of breakdown it is clear that the flow behaviour is again very similar. The low frequency response of the vortex breakdown location is still present, as is a frequency which can be associated with the helical mode. The occurrence of many more frequencies within the post-breakdown flow signal, may be attributed to the presence of many more smaller structures occurring in the flow for the USAFA $65^{\circ}$ solution, as shown in Figure 6. However, it is interesting to note that there is still little frequency content of the signal for frequencies close to or above a Strouhal number of 10 . 


\section{B. Resolution of DES Solutions}

The $70^{\circ}$ ONERA test case solution may be considered further by comparing the Glasgow results with existing USAFA DES results for the ONERA test case, available from the literature. $^{4,5,32,33}$ These results were obtained using the same geometry as the current investigation, however the trailing edge is blunt, similar to the experimental configuration. The Mach number used is also the same as in the experiments, $M=0.069$. The effect of grid refinement was considered and is detailed in Ref's. 4, 5, 32 and $33 . \quad$ In these investigations five grids of varying refinement were created and the solutions compared. Table 4 details the time step and grid size for the coarse, baseline and fine grids. The table also contains details of the nominal cell size, $\Delta_{o}$ in the focus region as described in Ref. 26. These features are also included for the fine grid used for the Glasgow DES results. Analysis was also carried out for a real fine grid with $10.6 \times 10^{6}$ cells and an adaptive mesh refinement (AMR) grid with $3.2 \times 10^{6}$ cell volumes, refined within the vortex core region.

It is interesting to note that the size of the cells in the focus region for the baseline grid, $\Delta_{o}$, is similar to that used for the Glasgow results, however the overall grid size of the USAFA grid is much smaller. This is due to the refinement in the region of interest having to be carried out to the far field for the structured grid. This increases the grid size and the relative computational expense. Therefore, the large structured grid is of comparable refinement to the baseline grid from the USAFA results. This is also clear from comparison of the flow solutions. Figure 10 shows instantaneous isosurfaces of vorticity for each of the grids, and highlights the increasing resolution of the flow structure with increasing grid refinement. From this, it is evident that the level of vortical structures captured by the Glasgow results is between the coarse and the baseline grid solutions.

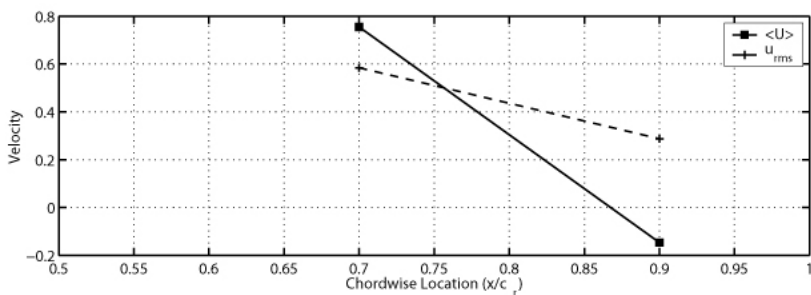

(a) Mean and RMS behaviour

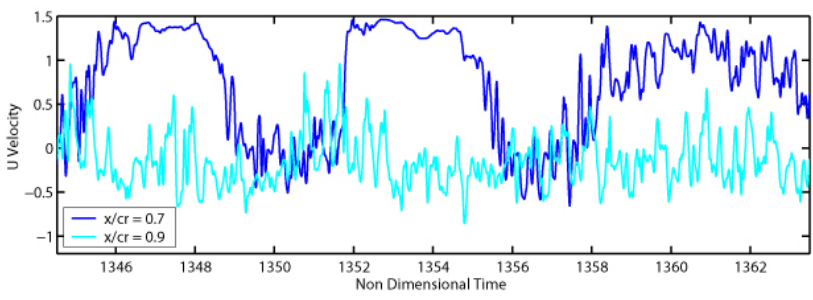

(b) Time history

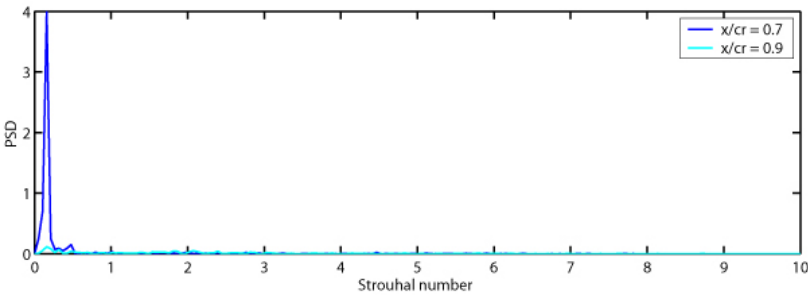

(c) PSD analysis

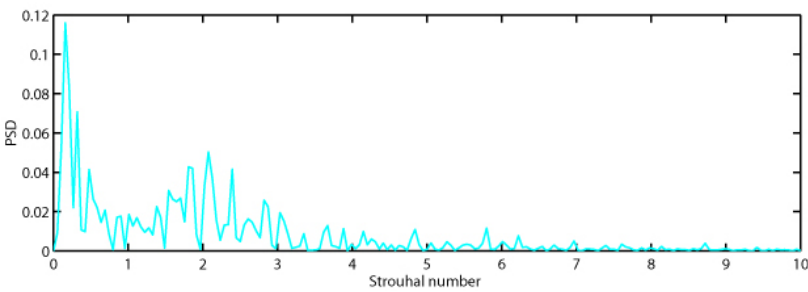

(d) PSD analysis with $x / c_{r}=0.70$ probe signal removed for clarity of frequency content of $x / c_{r}=0.90$ probe

Figure 9. Unsteady behaviour of non-dimensional $u$ velocity components at probes through vortex core region shown by Mean, RMS, time histories and PSD frequency plots for $65^{\circ} \mathrm{VFE}-2$ test case

Table 4. Details of grid features for USAFA grid study and comparison with current results

\begin{tabular}{cccc}
\hline & Cells & $\Delta_{o}$ & $\Delta \tau$ \\
\hline USAFA Coarse & $1.2 \times 10^{6}$ & 0.0065 & 0.00357 \\
USAFA Medium (baseline) & $2.7 \times 10^{6}$ & 0.0046 & 0.0025 \\
USAFA Fine & $6.7 \times 10^{6}$ & 0.0035 & 0.0018 \\
Glasgow Grid & $\sim 8 \times 10^{6}$ & 0.0055 & 0.0025 \\
\hline
\end{tabular}

To consider the grid resolution further it is necessary to examine the behaviour of the unsteady flow on the grid in this region. For an unsteady and turbulent flow it should be possible to see the fluctuations of 


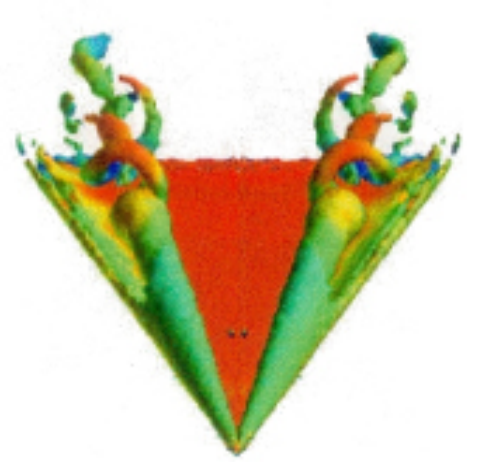

(a) Coarse $-1.2 \times 10^{6}$

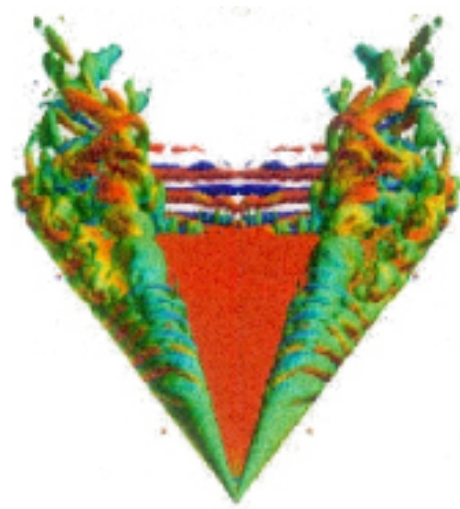

(d) Real Fine - $10.6 \times 10^{6}$

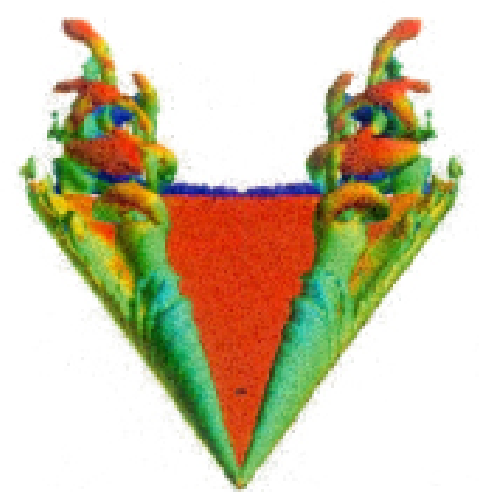

(b) Medium - $2.7 \times 10^{6}$

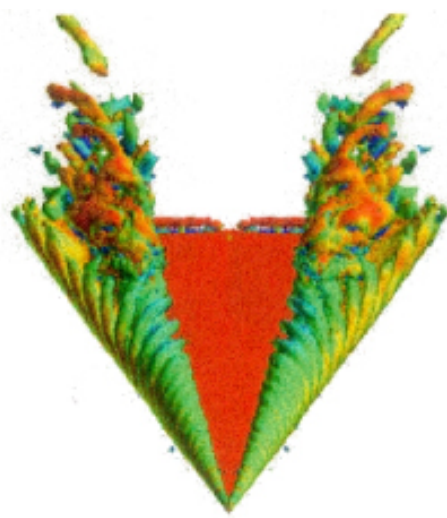

(e) AMR Grid - $3.2 \times 10^{6}$

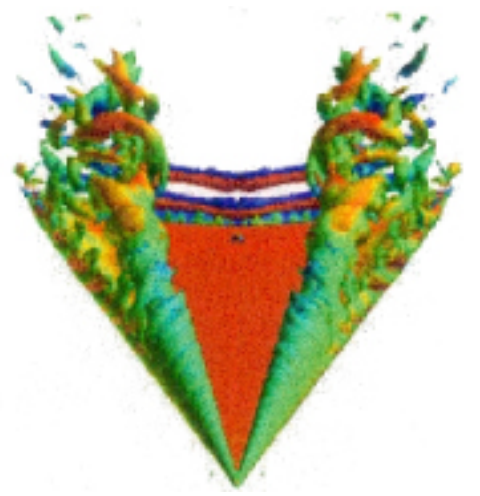

(c) Fine $-6.7 \times 10^{6}$

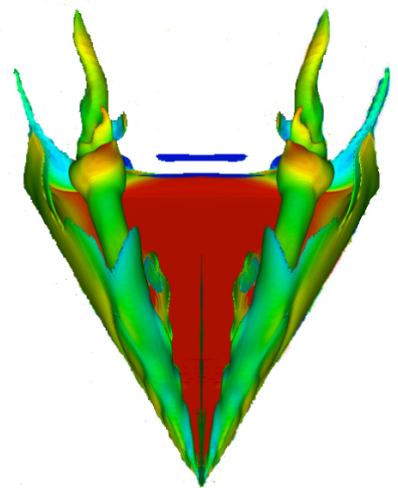

(f) Current Results - $\sim 8 \times 10^{6}$

Figure 10. Isosurfaces of Vorticity for various USAFA unstructured grids compared to Glasgow DES results on refined trailing edge structured grid. The number of cell volumes for each grid are given for comparison. $70^{\circ}$ ONERA test case. ${ }^{4}$

flow parameters on the grid. In LES, the grid is used as a spatial filter and, thus, the size of the cells are used to determine the spatial sample rate, in a similar manner to the use of the temporal sample rate, $\Delta \tau$. For structured grids, it is not practical to keep this sample rate uniform throughout the vortical region for delta wing geometries. However, for the Glasgow grid, in the region of interest close to the trailing edge the grid size is relatively constant at approximately $\Delta_{\max }=0.0055 c_{r}$. Using this as the sample rate, the maximum wavenumber of the spatial resolution can be determined for each grid. In Spalart's guide to DES grid generation, ${ }^{26}$ it is suggested that the minimum wavelength of a structure captured by a grid will be equal to five times the maximum grid size i.e. $5 \Delta_{\max }$. Using this as a guide, it can be calculated that the minimum non-dimensional wavelength captured by this grid in this region will be $0.0275 c_{r}$. This corresponds to a maximum non-dimensional wavenumber of approximately $\kappa=18$ and a minimum eddy size of $0.055 c_{r}$ due to the Nquist criterion.

To confirm this analysis, a 1-D slice through the vortex core region for each grid, was taken at a constant height above the wing surface $\left(z / c_{r}=0.1\right)$. Treating these slices in the the same way as a time trace, with $x / c_{r}$ being analogous to time, the data was analysed as before. A plot of $u$ velocity against $x / c_{r}$ is plotted for the flow downstream of breakdown for both results, this is shown in Figure 11(a). As the location of breakdown is different for each solution, the relative distance from the breakdown location is used. It is clear from this plot that there are more fluctuations of the velocity in the post-breakdown region for the USAFA results.

Performing a PSD analysis on this data allows the wavenumber content and resolved eddy sizes to be determined. Considering the results of this analysis, it was found that the dominant peak of both signals has a wavenumber of approximately $\kappa=0.5$. However, it was also found that energy exists at higher 
wavenumbers up until approximately $\kappa=18$ in Glasgow results, although very small. Most of the energy on the grid is found for wavenumbers less than 10, which is similar to the temporal analysis. This suggests that although smaller eddies are captured by the grid, they are very weak in comparison with the larger structures. Compared to these results, it was found that there is more energy in the larger wavenumbers for the USAFA results, however the maximum wavenumber resolved is still only approximately $\kappa=20$.

The physical size of these eddies can be considered from analysis of the non-dimensional wavelength of the signal, as shown in Figure 11(b). The wavelength is calculated as the inverse of the wavenumber. This plot is very interesting as it shows that the minimum wavelength captured on the grid is also close to $0.05 c_{r}$. However, the lowest clear peak is 0.11. Confirmation of the size of the captured eddies may be taken from the contours of $y$ vorticity (not shown) which clearly show structures with diameters of approximately 0.06 . It is evident from this analysis that the minimum eddy size is still approximately $5 \%$ of the root chord, which is relatively large, particularly with respect to the expected size of any small scale turbulent eddies, which would be less than $1 \% c_{r}$. For the USAFA results the maximum wavenumber found in the flow translates to a minimum wavelength of approximately $x / c_{r}=0.035$. Which is not significantly higher than the minimum wavelength of the Glasgow grid. Despite this similarity of minimum scales, more energy appears in the flow for all wavenumbers for the USAFA solution. This may be a consequence of the resolution of smaller scales which capture the energy transfer more accurately, due to a smaller sample rate and therefore less turbulence modelled on the grid.

To consider how this spatial under-resolution would affect the temporal resolution of the solution, it is possible to relate the frequency of the eddies to their wavelength, and therefore wavenumber, using the local velocity magnitude, as defined as,

$$
S t=u_{\text {local }} \kappa
$$

As the velocity at a given location will fluctuate in time, this relationship may only serve as a guide to the effect on the temporal resolution. However, in the post-breakdown the velocity is almost always less than the freestream. Therefore, the maximum non-

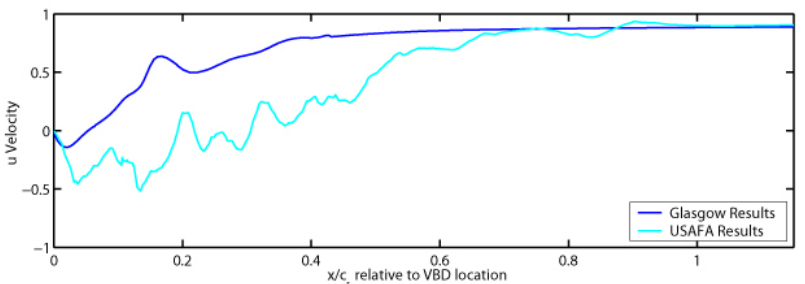

(a) $u$ velocity vs. $x / c_{r}$

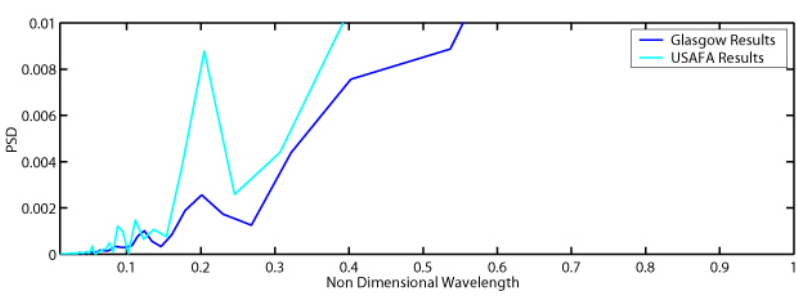

(b) Wavelength

Figure 11. $u$ velocity behaviour on slice through vortex core at $z / c_{r}=0.1$ relative to vortex breakdown location for both test cases

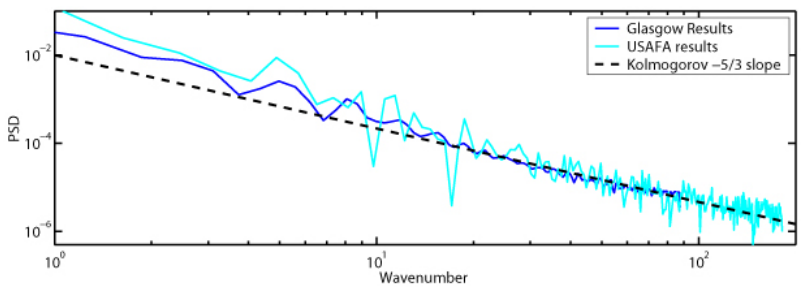

(a) Spatial - PSD vs. $\kappa$ compared to Kolmogorov $-5 / 3$ slope

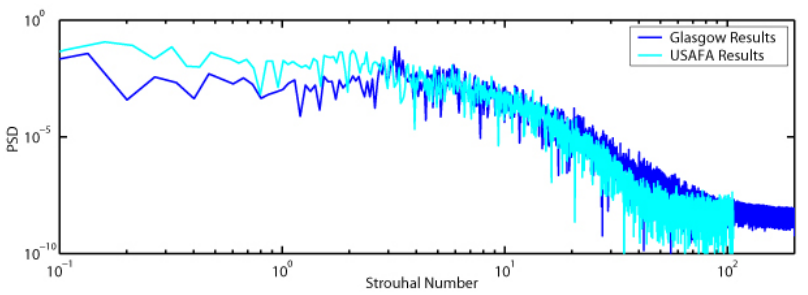

(b) Temporal - PSD vs. St for $u$ velocity from probe in postbreakdown flow at $x / c_{r}=1.00$

Figure 12. Spatial and temporal comparisons of USAFA and Glasgow DES results dimensional frequency resolved on the grid will be less than 18 for the Glasgow results and less than 20 for the USAFA results. The ability of the spatial and temporal sampling rate to capture the turbulence may be determined by considering a log-log plot of the PSD analysis. Figure 12 shows the results of this analysis for both test cases. The spatial resolution can be compared to the Kolmogorov $-5 / 3$ slope, which describes the theoretical behaviour of the energy within the turbulence for the inertial subrange. The temporal comparison is created from the non-dimensional $u$ velocity at the post-breakdown probe location detailed previously. It is 

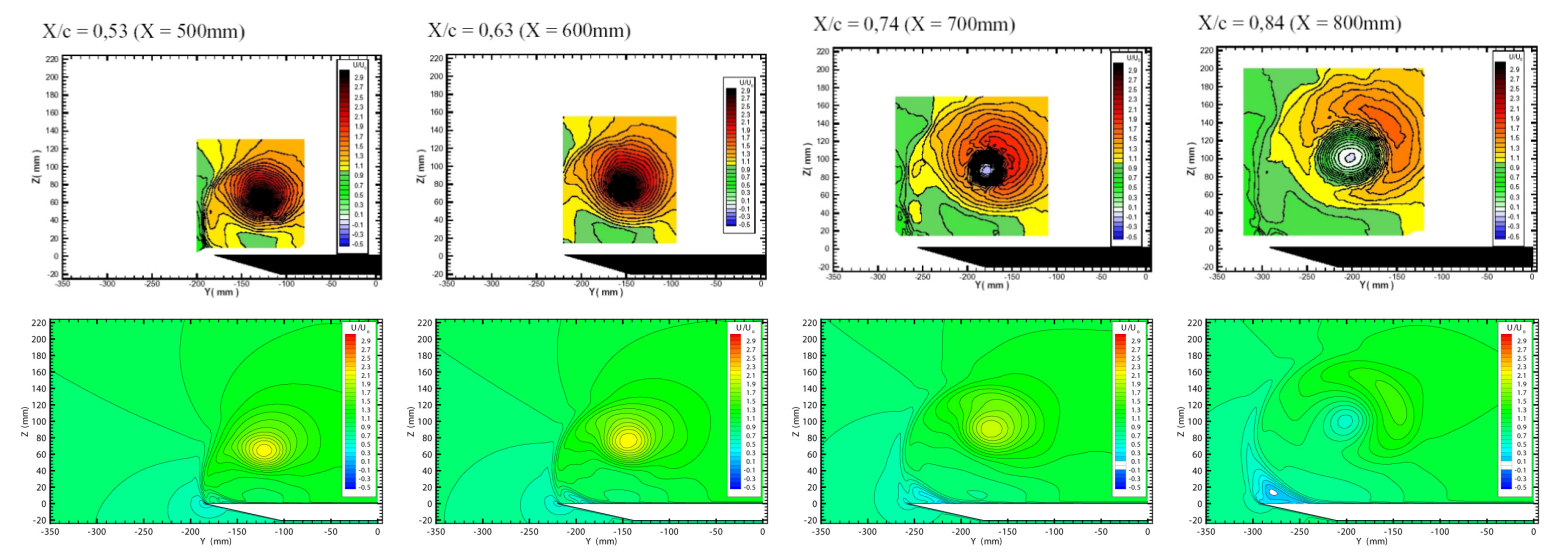

Figure 13. Time-averaged $u$ velocity results from Mitchell's experiment compared to mean computational results from Glasgow DES solutions for $70^{\circ}$ ONERA test case
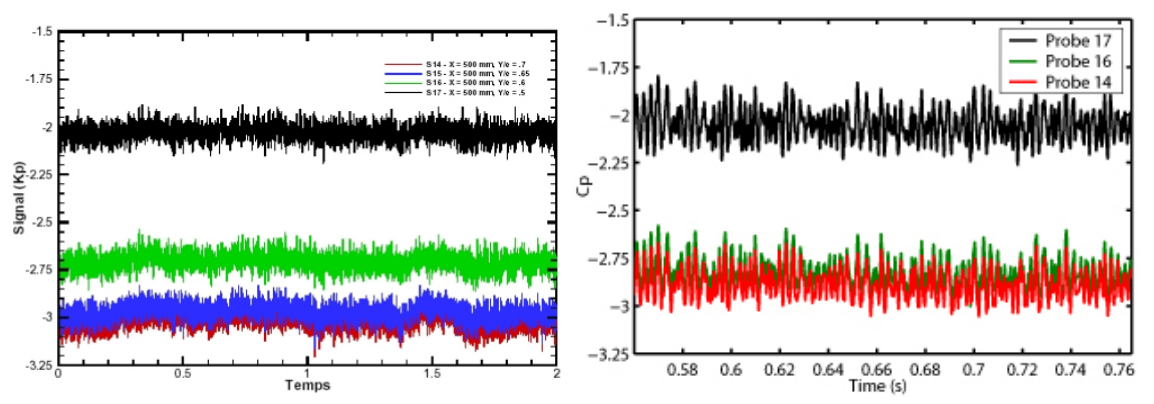

(a) $x / c_{r}=0.53$

Figure 14. Time histories of unsteady pressure from Glasgow DES solutions for the $70^{\circ}$ ONERA test case compared to corresponding experimental data ${ }^{11}$

evident from both plots that the general frequency behaviour is very similar between the two sets of solutions. Although the USAFA grid exhibits slightly more energy at higher wavenumbers in the spatial comparison, the temporal comparison is nevertheless very similar, with the same gradient to higher frequencies being present. Therefore, despite the higher grid resolution of the USAFA solution demonstrated by the smaller scale structures found in the post-breakdown flow and the greater frequency content, it may be stated that a similar level of turbulence is captured by each solution.

\section{Validation of DES Results}

Initial comparisons may be made with the time-averaged results obtained from the experiments carried out by Mitchell. ${ }^{11}$ The time-averaging process used in the experiments is akin to calculating the stationary mean of the flow and does not take the unsteady mean flow into account. Instantaneous full domain flow solutions could not be used to compare with this data. Therefore, a stationary mean was calculated from 100 time steps, over a total time of $\tau=1$, which gives a sample rate of $\Delta \tau=0.01$. This provides a relatively small period over which to average, however the amount of data needed to perform a full mean calculation over the entire calculation was prohibitive. Due to this, the comparisons should be treated with caution, but should be sufficient for the purposes of validation of the basic flow behaviour. igure 13 show contours of the non-dimensional mean $u$ velocity for each of the chordwise stations for both the experiment and the mean computational flow. It is clear that for the experimental data breakdown occurs upstream of the $x / c_{r}=0.74$ position, as at this location reversed flow is found. Indeed, from the investigation it was found that the mean position of breakdown occurred at approximately $x / c_{r}=0.65$. Considering the DES results, it is clear that the location of breakdown is quite different, with reversed flow not being predicted for any 


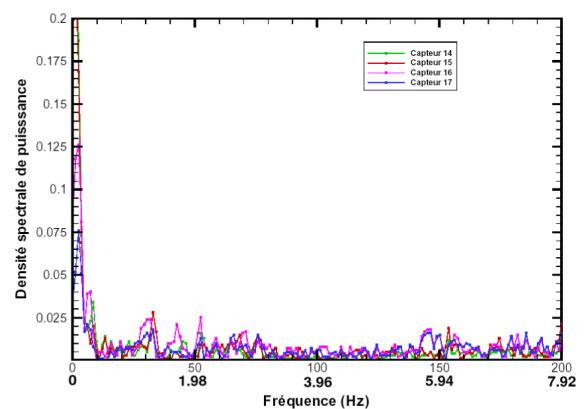

(a) Experiment - $0.15 c_{r}$ upstream of breakdown

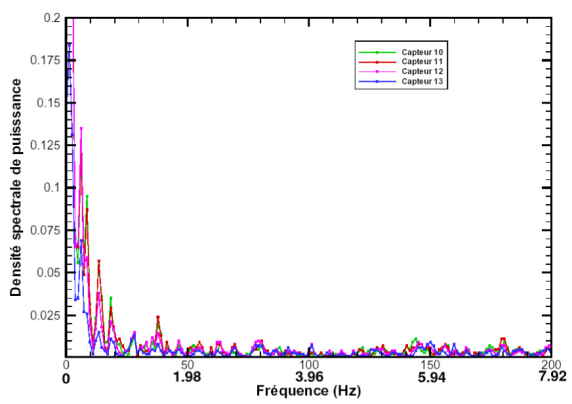

(c) Experiment - 0.02c $c_{r}$ upstream of breakdown

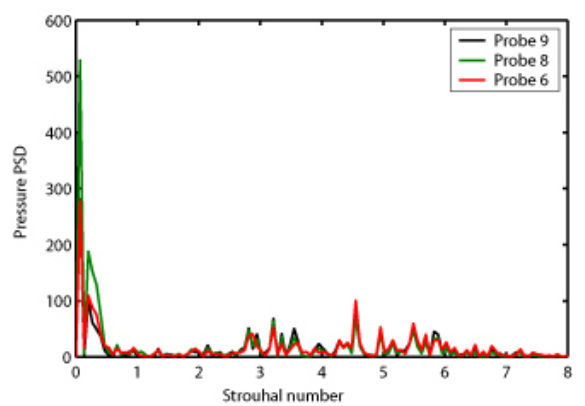

(b) CFD - 0.14c $c_{r}$ upstream of breakdown

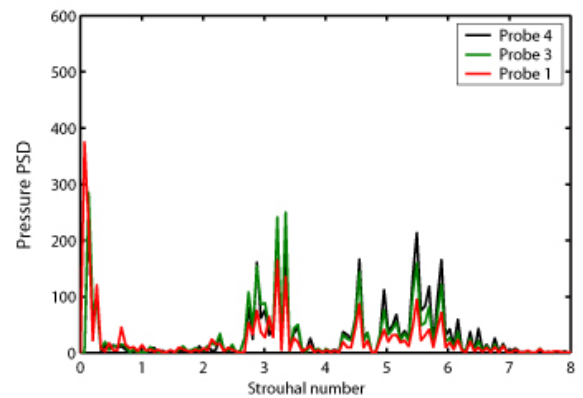

(d) CFD - 0.04c $c_{r}$ upstream of breakdown

Figure 15. Power spectral density plots of unsteady pressure probe data from Glasgow DES solution for $70^{\circ}$ ONERA test case compared to experimental results ${ }^{11}$

of the slices. The mean breakdown location is found to occur downstream at approximately $x / c_{r}=0.86$. The discrepancy of mean vortex breakdown location may be due to many factors such as imperfections on the experimental model due to the sting fitting, or the levels of turbulent eddy viscosity predicted in the computational results. The grid study and time step study, to be detailed, show that the predicted location of breakdown do not change significantly with any change in grid density or time step refinement, thus the DES calculations are consistent. This discrepancy of location should also be kept in mind when considering the unsteady nature of the flow.

The maximum axial velocity of the vortex core prior to breakdown was approximately $4 U_{\infty}$. The mean predicted value from the Glasgow results, is found to be considerably less and is given as approximately $2.2 U_{\infty}$. This is consistent with the findings of the AVT-080 task group, where a number of calculations were performed for this test case using various CFD solvers, techniques and grids. The axial velocity was not found to be accurately predicted for any of the cases and it was concluded that the grid refinement at the vortex core was not sufficient.

To consider the unsteady nature of the flow, readings were taken from 17 Kulite pressure transducers on the surface of the wing. These probes were situated at constant spanwise locations on the wing, $y / s=0.5$, 0.6 and 0.7 at each streamwise location. The unsteady pressure coefficient time histories for $x / c_{r}=0.53$ are shown in Figure 14 along with the comparable DES results, to provide an example of the comparison. From this trace, it is clear that the mean pressure decreases with outboard movement on the wing. This suggests that the vortex core sits either above or close to the $y / s=0.7$ position. In the computational results, the vortex core is also found to close to this location. The mean and magnitude of the oscaillations are in reasonably good agreement.

To consider the frequency content of the signals, a PSD analysis was performed on each signal. Due to the difference in location of vortex breakdown it may not be possible to make direct comparisons between 
the frequency responses for a given chordwise location. Therefore, the non-dimensional distance from the breakdown location was considered. As the mean breakdown location is $x / c_{r}=0.65$ in the experiments, the $x / c_{r}=0.53$ station is $0.15 c_{r}$ upstream and the $x / c_{r}=0.63$ station is $0.02 c_{r}$ upstream of this location. Similarly, for the computational results, the $x / c_{r}=0.74$ station is $0.14 c_{r}$ upstream and the $x / c_{r}=0.84$ station is $0.04 c_{r}$ upstream of breakdown. Therefore, for validation these locations are compared and are shown in Figure 15.

The plots taken from Mitchell's work have been altered slightly to show the corresponding non-dimensional frequencies for comparison. From the experimental plots, the flow behaviour is dominanted by a low frequency oscillation, which occurs at approximately $S t=$ 0.07. There is evidence of some higher frequency broadband content, however this has relatively low power in comparison. Comparing this to the DES results, it is clear that the agreement is reasonable, with the low dominant frequency occurring at $S t=0.07$ and the higher frequency content focusing around $S t=3-5$. However, these higher frequencies have more power in the CFD results, particularly close to breakdown and do not have such a broadband appearance. From the unsteady analysis performed on the DES results these frequencies were attributed to the helical mode winding and possible shear layer behaviour. However, evidence of the upstream influence of these phenomenon on the surface of the wing is not found in the experiment. Although a dominant frequency of approximately $S t=3.25-4.25$ is found downsteam of breakdown.

From the unsteady analysis, it was determined that a low frequency response exists in the flow close to breakdown of $S t=0.07$. This was attributed to the unsteady oscillation of the breakdown location. It was found in the experiment, that this behaviour occurred at a non-dimensional frequency of

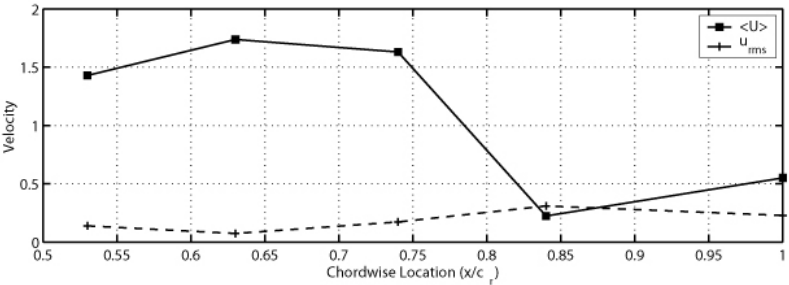

(a) Mean and RMS behaviour

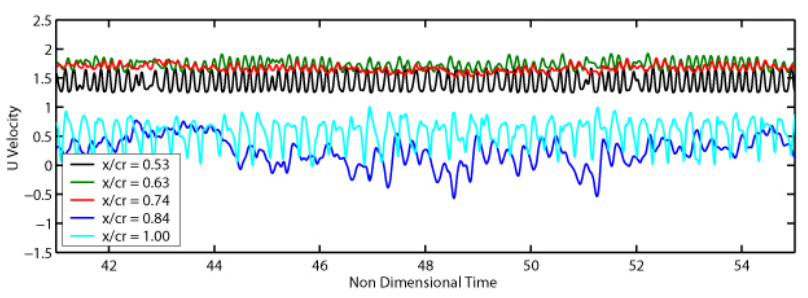

(b) Time history

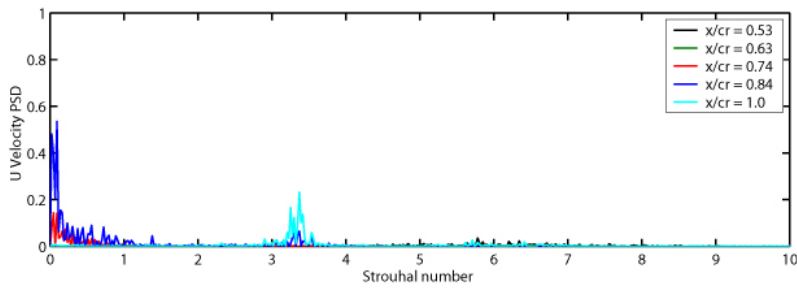

(c) PSD analysis

Figure 16. Unsteady behaviour of non-dimensional $u$ velocity at probes through vortex core region shown by shown by Mean, RMS, time histories and PSD frequency plots for $k-\omega$ with $P_{\omega}$ enhancer model, $\Delta \tau=0.01$ for $70^{\circ}$ ONERA test case $S t=0.043$ with an amplitude of oscillation of approximately $15 \%$ root chord. This corresponds to a location $x / c_{r}=0.6-0.75$ for the left hand side and $x / c_{r}=0.65-0.8$ for the right hand side vortex. Comparatively, the computational results predict an oscillation with an amplitude of approximately $6 \%$ root chord. This under-prediction of the amplitude may be due to the symmetric assumption as in the experiment there may be interaction between the behaviour of the two leading edge vortices.

\section{Evaluation of URANS Results}

\section{A. Unsteady Behaviour of URANS Solutions}

Figure 16 shows the unsteady flow behaviour in the streamwise direction for the $k-\omega$ with $P_{\omega}$ enhancer model. For this case, it is found that the probes at $x / c_{r}=0.53,0.63$ and 0.74 are upstream of breakdown, with all probes sitting above the vortex core axis. The probe at $x / c_{r}=0.53$ sits within the shear layer and the probe at $x / c_{r}=0.63$ sits in the region between the vortex core and the shear layer. As breakdown occurs at $x / c_{r}=0.80$, the probes at $x / c_{r}=0.84$ and 1.0 are within the post-breakdown region. 
The time histories show that, upstream of breakdown, the fluctuations of the velocity have relatively low amplitude, compared with those downstream of breakdown. At $x / c_{r}=0.53$, the trace exhibits a slightly larger amplitude and higher frequency than for the probes closer to the vortex core, due to its location in the shear layer. This may be due to the presence of shear layer instabilities. At $x / c_{r}=0.84$, the velocity time history shows a large amplitude, low frequency oscillation consistent with the fluctuation of vortex breakdown location. It is evident that the breakdown location passes over this position as the velocity decreases below zero, suggesting recirculating flow. A higher frequency clearly exists in this signal also. Further downstream, at the trailing edge, the low frequency behaviour appears to have disappeared and a higher frequency remains.

From analysis of the frequency content of the time traces, a number of dominant flow frequencies can be identified. It is evident that there are two main dominant frequencies. At $x / c_{r}=0.84$, the dominant frequencies in the signal appear to be centred around $S t=0.07$, which has previously been identified as the dominant frequency for the oscillation of vortex breakdown. A second smaller peak is also evident at approximately $S t=3.25$ and is associated with the helical mode instability and winding and corresponds to the higher frequency mentioned above. Downstream of breakdown, this frequency is dominant. With a closer look at the PSD analysis,

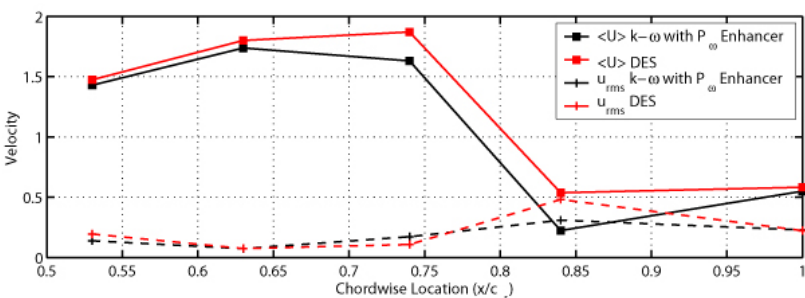

(a) Mean and RMS behaviour

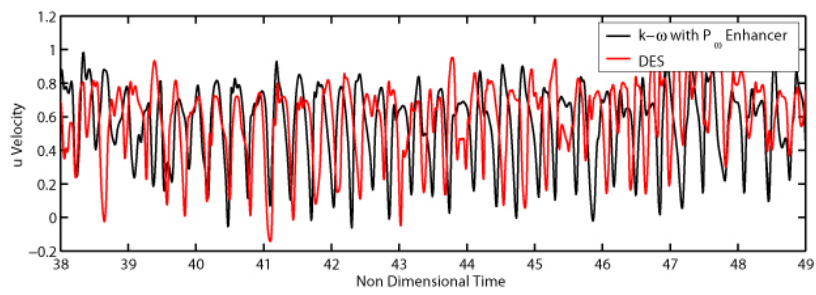

(b) Time history

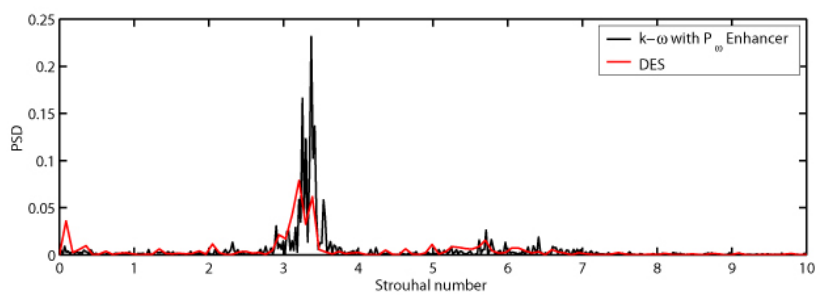

(c) PSD analysis

Figure 17. Unsteady behaviour of non-dimensional $u$ velocity at probes through vortex core region shown by shown by Mean, RMS, time histories and PSD frequency plots for $k-\omega$ with $P_{\omega}$ enhancer model, $\Delta \tau=0.01$ and DES solutions, $\Delta \tau=0.0025$ on the Fine grid for the $70^{\circ}$ test case further frequencies may be determined in the signals upstream of breakdown. It was found that the effect of the oscillation of breakdown location was also mildly felt upstream of breakdown at $x / c_{r}=0.74$. At $x / c_{r}=0.53$, the higher frequencies associated with the time trace described before were found to correspond to $S t \approx 5-8$, which is within the possible frequency range for shear layer instabilities.

\section{B. DES/URANS Comparison}

To determine the ability of the URANS calculations to capture the main unsteadiness of the flow, the unsteady URANS results were compared to the DES solutions detailed previously. For the $70^{\circ}$ ONERA test case, the DES and URANS solutions were obtained on the same computational grid to allow fair comparison. The analysis involves comparing the results shown in Figure 16 to the DES results in Figure 7. Direct comparisons of the mean and RMS $u$ velocity and unsteady analysis of the probe at $x / c_{r}=1.00$ are shown in Figure 17.

From consideration of the mean velocities, it is clear that the behaviour is very similar, however the URANS model consistently predicts a lower mean than the DES solution. The RMS values are also very similar, with the exception of the $x / c_{r}=0.84$ location where the RMS velocity is higher for the DES model. At this location the amplitude of the low frequency response is more pronounced for the DES solution. This due to differences in the mean predicted breakdown location, which is $x / c_{r}=0.8$ for the URANS solution 
and $x / c_{r}=0.86$ for the DES results. However, at the trailing edge the RMS and mean values are very close. This is also evident from the time histories of the $u$ velocity at the trailing edge which shows good agreement between the results.

Considering the frequency content for each solution, it is evident that the dominant frequencies are similar. The URANS model predicts the helical mode winding at $S t=3.25$ which is the same for the DES results and the vortex breakdown oscillation frequencies also coincide. Again, this is clear from the PSD plot shown in Figure 17 at the trailing edge. The dominant frequencies are in clear agreement, although differences in their power are evident. Similarly, higher frequencies in the signal between $S t=5-7$ are also predicted by both turbulence treatments. Despite similar frequencies being present it is clear that the levels of energy are larger for the URANS results. This may be due to differences in the length of signal caused by the different time steps used.

From these analyses and comparisons it is evident that the URANS models can predict the characteristic unsteady behaviour of the vortical flow both preand post-breakdown. However, before further conclusion are made, the sensitivity of both the DES and URANS solutions to grid refinement and time step refinement will be detailed.

\section{Sensitivity of Solutions to Numerics}

Using the $70^{\circ}$ ONERA test case, the effect of grid refinement for both the DES and URANS and temporal refinement for the DES calculations were considered.

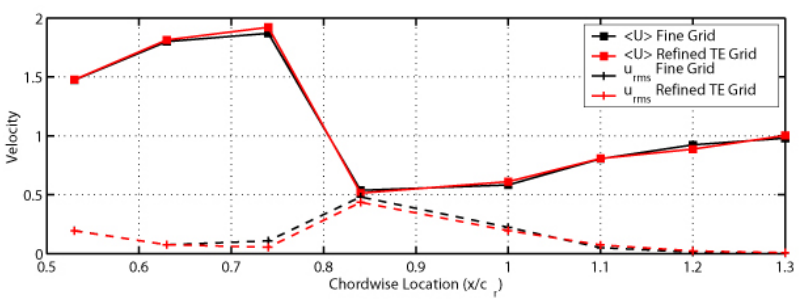

(a) Mean and RMS behaviour

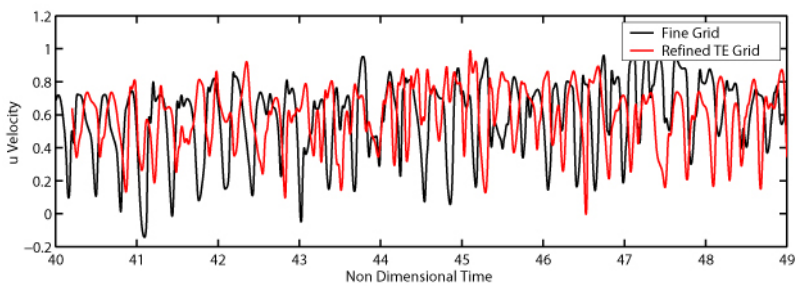

(b) Time history

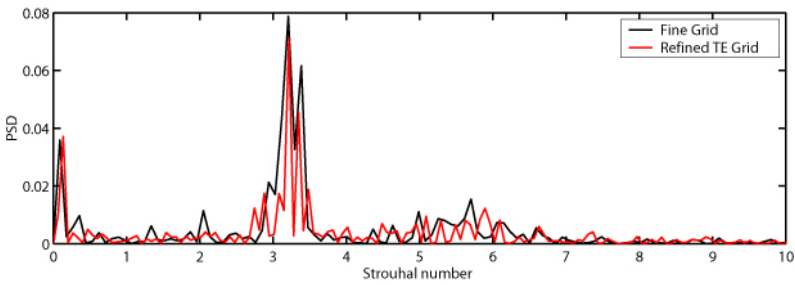

(c) PSD analysis

Figure 18. Unsteady behaviour of non-dimensional $u$ velocity shown by Mean and RMS velocities through the vortex core region and time histories and PSD frequency plots for the probe at $x / c_{r}=1.00$ for the Fine grid and the refined TE grid for the $70^{\circ}$ test case.

\section{A. Effect of Grid Refinement}

\section{DES}

From the fine grid results it was noted that the turbulence in the DES solutions may have dissipated too soon beyond the trailing edge due to the large stretching ratio of the grid points in this region. To investigate this, a grid was created which had greater refinement in this region as shown in Figure 4. The maximum cell dimension, $\Delta_{o}$, in the trailing edge region was the same for both grids. However, the this region was extended further downstream due to the grid refinement in the streamwise direction. The calculations were both performed using a non-dimensional time step of $\Delta \tau=0.0025$.

The unsteady analysis of both solutions are shown in Figure 18 showing the mean and RMS velocities and the unsteady probe behaviour at the trailing edge. From analysis of the mean and RMS $u$ velocities it is clear that the behaviour at the locations through the vortex core region and downstream of the trailing edge are very similar. It is found from analysis of the mean flow domain that the location of vortex breakdown is also the same for the two grids. Considering the unsteady behaviour at the trailing edge and it is evident that, although there are differences in the time histories, overall the mean location and amplitude of the signal 
are very similar. This is also true when considering the frequency content of the signals. All the dominant frequencies of the flow identified before are captured by both solutions and the agreement is good for both frequency and mangitude. Therefore, it was concluded that the refinement of the grid at the trailing edge is not a important feature for the DES calculations for capturing the turbulence downstream of breakdown.

\section{URANS}

URANS flow solutions are only dependent on grid refinement for numerical accuracy, which improves as the size of the cells decrease. To consider the effect of the grid refinement, calculations were performed using the $k-\omega$ with $P_{\omega}$ enhancer model at a time step of $\Delta \tau=0.01$ for coarse and fine grids.

The mean and RMS values of the $u$ velocity are shown in Figure 19. From this plot, it is clear that the vortex breakdown occurs upstream of the $x / c_{r}=0.74$ location for the coarse grid. Indeed, analysis of the flow behaviour shows that the location of vortex breakdown on the wing is different for the two grids. From the mean flow solutions, the location of vortex breakdown was found to occur at approximately $x / c_{r}=0.72$ for the coarse grid and $x / c_{r}=0.80$ for the fine results. This difference in location, is most likely to be due to the differences in resolution of the vortex core behaviour. Upstream and downsteam of this location, the mean values of both solutions are in good agreement.

Considering the RMS velocity, it is found that upstream of breakdown the the levels of unsteadiness are very similar for both grids. This is also true at $x / c_{r}=0.74$, despite vortex breakdown having occurred upstream of this location for the coarse grid. Further downstream, the level of unsteadiness has increased for the fine grid solutions due to vortex breakdown occurring. Downstream of this location

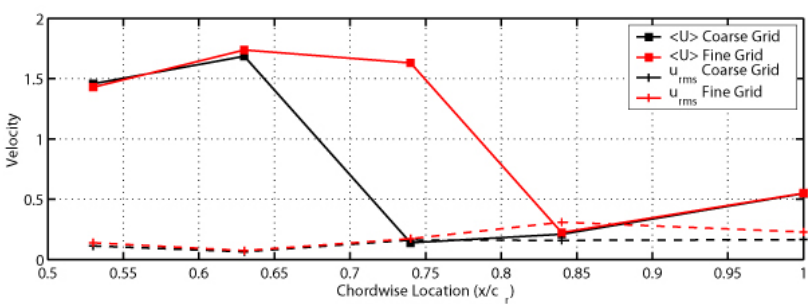

(a) Mean and RMS behaviour

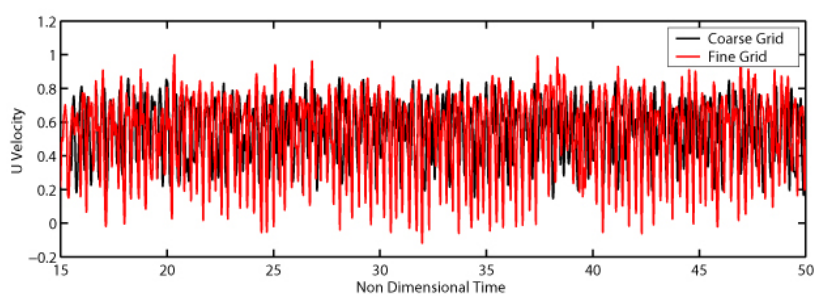

(b) Time history

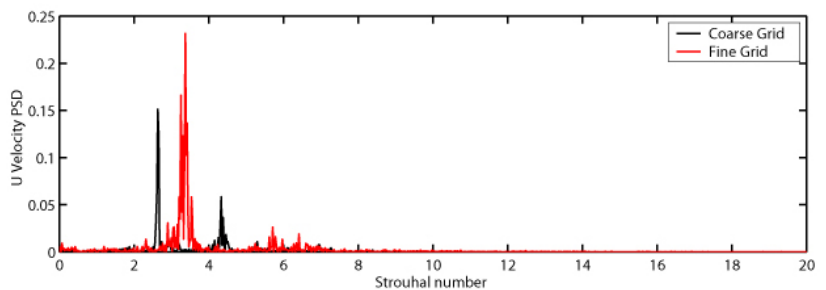

(c) PSD analysis

Figure 19. Unsteady behaviour of non-dimensional $u$ velocity shown by Mean and RMS velocities through the vortex core region and time histories and PSD frequency plots for the probe at $x / c_{r}=1.00$ for the $k-\omega$ with $P_{\omega}$ enhancer model on coarse and Fine grids for the $70^{\circ}$ test case the levels drop off for the coarse grid and it is clear that the fine grid exhibits greater unsteadiness in the post-breakdown region.

To further analyse the unsteadiness in the post-breakdown region, a single probe situated above the trailing edge is considered for both cases and is also shown in Figure 19. It is clear that at this location, the mean velocities are virtually identical for both grids, but that the RMS velocities and therfore the levels of unsteadines are quite different. From the time history, the most noticeable difference between the signals is that the fine grid solutions gives a signal with a greater amplitude than the coarse grid, in agreement with the RMS values discussed above. Considering the frequency content of the signals, it is clear that the behaviour is quite different. The coarse grid predicts two dominant frequencies at approximately $S t=2.6$ and 4.25 with a much smaller peak evident at $S t \approx 5.2$, a harmonic of the first dominant peak. The fine grid, however, only predicts one dominant peak at approximately $S t=3.25$ and some higher frequency content at $S t=4.5-7$. For the fine grid, has already been determined that this dominant frequency is associated to the helical mode instability. However, the source of the two peaks in the coarse grid results is not so obvious. It is quite likely that they are also related to the rotation of the vortex breakdown winding, however 
the behaviour is quite different to that expected. From this analysis, it is clear that the fine grid produces results with greater resolution of the flow features and a more accurate prediction of the unsteady nature of the flow, particularly downstream of breakdown.

\section{B. Effect of Time Refinement for DES solutions}

A similar analysis was carried out for the DES calculations for the $70^{\circ}$ test case to consider the effect of time step refinement on the prediction of the unsteady behaviour. The fine grid was used for this study with nondimensional time steps of $\Delta \tau=0.01,0.005$ and 0.0025. An similar analysis as before, was performed on the unsteady probe signals and the results are shown in Figure 20. From this analysis, it is clear that changing the time step if the solution has an overall effect on the mean and RMS velocities of the predicted flow. However, upstream at $x / c_{r}=0.53$ and 0.63 the predicted values are all in good agreement, are not affected by temporal resolution. The mean breakdown location is found to differ between each of the solutions and occurs at $x / c_{r}=$ 0.88 for $\Delta \tau=0.01, \quad x / c_{r}=0.90$ for $\Delta \tau=0.005$ and $x / c_{r}=0.86$ for $\Delta \tau=$ 0.0025. Close to breakdown at $x / c_{r}=$ 0.84 , the differences between the mean velocity results are most pronounced, most likely due the differences in mean breakdown location. However, the RMS values appear to be very similar at this location and downstream.

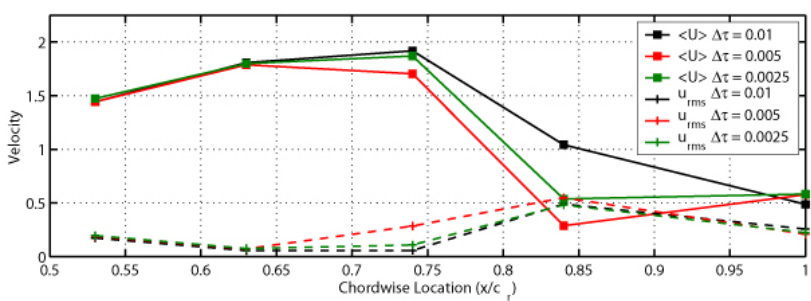

(a) Mean and RMS behaviour

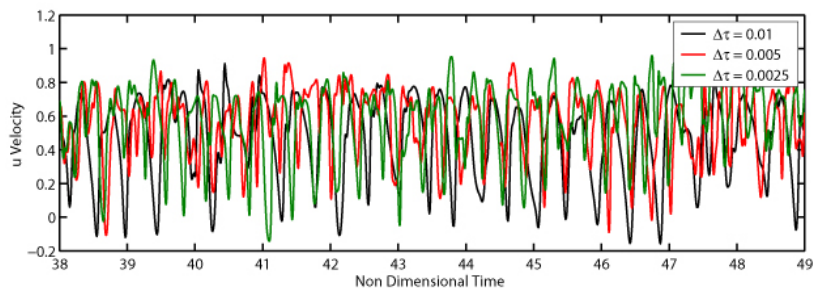

(b) Time histories

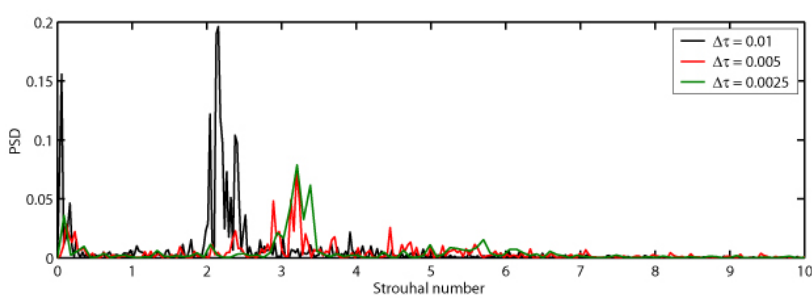

(c) PSD analysis

Considering a single probe in the flow above the trailing edge, as before, comparisons of the unsteady behaviour can be made. At this location it is found that the mean and RMS values are is good agreement, and from the time trace it is clear that the amplitude of the signals also agrees overall. How-

Figure 20. Unsteady behaviour of non-dimensional $u$ velocity shown by Mean and RMS velocities through the vortex core region and time histories and PSD frequency plots for the probe at $x / c_{r}=1.00$; Glasgow DES solutions for the Fine grid at $\Delta \tau=0.01,0.005$ and 0.0025 for the $70^{\circ}$ test case ever, it is clear that the signal behaviours are different, particularly the signal from the $\Delta \tau=0.01$ solution which clearly exhibits a lower frequency oscillation that the other two signals. From the PSD analysis of these results, the dominant frequencies are determined and again the differences between the coarsest time step and the other results is striking. Both the $\Delta \tau=0.005$ and 0.0025 results show the dominant frequency to occur at approximately $S t=3.25$. However, for $\Delta \tau=0.01$ this is lower at approximately $S t=2.25$. Similarly for the vortex breakdown location frequency, the signal for $\Delta \tau=0.01$ exhibits a frequency lower than the $S t=0.07$ peak found for the other two results. The agreement between the $\Delta \tau=0.005$ and 0.0025 unsteady behaviour is very good. Thus, it may be suggested that the time step has converged.

To consider the appropriate time step for use in the DES calculations for this grid, the time step guide recommended by Spalart ${ }^{26}$ was used. This uses the nominal grid size in the region of interest to define a guideline time step for a particular grid. The relationship used is defined in Equation 15.

$$
\Delta \tau=\frac{\Delta_{o}}{U_{\max }}
$$

where $U_{\max }$ is the maxiumum velocity in the flow - a multiple of $U_{\infty}$. As the nominal value of $\Delta_{\max }$ is 
approximately 0.0055 for the Fine grid and taking $U_{\max }=2.5$, a guideline time step would be $\Delta \tau=0.0036$ which is slightly larger than the time step used for the investigation.

\section{Conclusions}

From the analysis of the DES solutions, it is clear that there are a number of dominant flow features, which occur in the flow above a slender delta wing. The main features found relate to the helical mode instability of vortex breakdown, the motion of vortex breakdown and possible shear layer structures. Each phenomenon causes different frequencies to be dominant at different locations in the flow, upstream, at and downstream of breakdown. Through the analysis of the results, it was shown that the frequencies found in the flow are all relatively low and the structures which occur appear to be periodic in nature. This lead to the conclusion that these features are coherent characteristic of the mean flow and not due to turbulence.

The resolution of the DES results was considered and it was found that only a small proportion of turbulence is resolved on the grid and it was shown that little improvement is made by refining the trailing edge region. From this analysis, it may be proposed that the grid size needed to fully capture the turbulence for delta wing flows remain prohibitive to a widespread use of DES, particularly for structured grids. This is due to the resulting computational cost of such fine grids and corresponding time steps. However, it was shown from validation with experimental results that the under-resolution of turbulence may not be detrimental to the prediction of the main features of the flow. Particularly for the use in predicting buffet for engineering flows.

As the dominant frequencies exhibited by the flow were low, it was proposed that URANS turbulence models could be used to adequately model the flow behaviour for the purposes of predicting buffet as the higher frequencies associated with turbulence are unlikely to have a considerable effect on the wing. From analysis of the URANS solutions it was found that indeed the results were capturing the main frequencies with significant accuracy, compared to the DES results, for the same grid. Thus, for considerably less computational expense these features could be predicted well.

\section{Acknowledgments}

Thanks go to BAE Systems for the CASE Sponsorship which funded this research.

\section{References}

\footnotetext{
${ }^{1}$ Gursul, I., "Review of Unsteady Vortex Flows over Slender Delta Wings," Journal of Aircraft, Vol. 42, No. 2, March-April 2005, pp. 299-319.

${ }^{2}$ Nelson, R. C. and Pelletier, A., "The Unsteady Aerodynamics of Slender Wings and Aircraft undergoing Large Amplitude Maneuvers," Progress in Aerospace Sciences, Vol. 39, 2003, pp. 185-248.

${ }^{3}$ Spalart, P. R., Jou, W.-H., Strelets, M., and Allmaras, S. R., "Comments on the Feasibility of LES for Wings and on a Hybrid RANS/LES Approach," Advances in DNS/LES, 1st AFSOR International Conference on DNS/LES, AFOSR, August 1997.

${ }^{4}$ Mitchell, A. M., Morton, S. A., Forsythe, J. R., and Cummings, R. M., "Analysis of Delta-Wing Vortical Substructures Using Detached Eddy Simulation," AIAA Journal, Vol. 44, No. 5, May 2006, pp. 964-972.

${ }^{5}$ Morton, S. A., "High Reynolds Number DES Simulations of Vortex Breakdown over a $70^{\circ}$ delta wing," 21st Applied Aerodynamics Conference, AIAA Paper 2003-4217, June 2003.

${ }^{6}$ Morton, S. A., Forsythe, J., Mitchell, A., and Hajek, D., "DES and RANS Simulations of Delta Wing Vortical Flows," 40th AIAA Aerospace Sciences Meeting and Exhibit, AIAA Paper 2002-0587, January 2002.

${ }^{7}$ Allan, M., A CFD Investigation of Wind Tunnel Interference on Delta Wing Aerodynamics, Ph.D. thesis, University of Glasgow, October 2002.

${ }^{8}$ Allan, M. R., Badcock, K. J., Barakos, G. N., and Richards, B. E., "Wind Tunnel Interference Effects on Delta Wing Aerodynamics Computational Fluid Dynamics Investigation," Journal of Aircraft, Vol. 42, No. 1, January-February 2005, pp. 189-198.

${ }^{9}$ Görtz, S., Realistic Simulations of Delta Wing Aerodynamics Using Novel CFD Methods, Ph.D. thesis, Aeronautics and Vehicle Engineering, KTH, June 2005.

${ }^{10}$ Mitchell, A. M., "Experimental Database Selected for RTO/AVT Numerical and Analytical Validation and Verification: ONERA 70 Delta Wing," 21st Applied Aerodynamcis Conference, AIAA 2003-3941, June 2003.
} 
${ }^{11}$ Mitchell, A. M., Caracterisation et Controle de L'eclatement Tourbillonnaire sur une Aile Delta aux Hautes Incidences, Ph.D. thesis, L'Universite Paris 6, July 2000.

${ }^{12}$ Mitchell, A. M., Molton, P., Barberis, D., and Delery, J., "Vortical Substructures in the Shear Layers Forming Leading Edge Vortices," 19th Applied Aerodynamics Conference, AIAA 2001-2424, June 2001.

${ }^{13}$ Mitchell, A. M., Molton, P., Barberis, D., and Delery, J., "Characterization of Vortex Breakdown by Flowfield and Surface Measurements," 38th Aerospace Science Meeting and Exhibit, AIAA Paper 2000-0788, January 2000.

${ }^{14}$ Mitchell, A. M. and Molton, P., "Vortical Substructures in the Shear Layers Forming Leading Edge Vortices," AIAA Journal, Vol. 40, No. 8, September 2002, pp. 1689-1692.

${ }^{15}$ Hummel, D. and Redeker, G., "A New Vortex Flow Experiment for Computer Code Validation," Proceedings of the RTOAVT Symposium on "Advanced Flow Management: Part A - Vortex Flows and High Angle of Attack for Military Vehicles" RTO-MP-069(I), NATO RTO, 2001, pp. 8.1-8.32.

${ }^{16} \mathrm{Chu}$, J. and Luckring, J. M., "Experimental Surface Pressure Data obtained on a $65^{\circ}$ Delta Wing across Reynolds Number and Mach Number ranges: Volume 1 - Sharp Leading Edge," NASA Technical Memorandum 4645, NASA Langley Research Centre, February 1996.

${ }^{17}$ Badcock, K. J., Richards, B. E., and Woodgate, M. A., "Elements of Computational Fluid Dynamics on Block Strucured Grids using Implicit Solvers," Progress in Aerospace Sciences, Vol. 36, 2000, pp. 351-392.

${ }^{18}$ Roe, P. L., "Approximate Riemann Solvers, Parameter Vectors and Difference Schemes," Journal of Computational Physics, Vol. 43, 1981, pp. 357-372.

${ }^{19}$ Osher, S. and Solomon, F., "Upwind Difference Schemes for Conservation Laws," Mathematics of Computing, Vol. 38, 1982, pp. 339-374.

${ }^{20}$ Strang, W. Z., Tomaro, R. F., and Grismer, M. J., "The Defining Methods of Cobalt: A Parallel, Implicit, Unstructured Euler/Navier-Stokes Flow Solver," AIAA Paper 99-0786, January 1999.

${ }^{21}$ Wilcox, D. C., Turbulence Modelling for CFD, DCW Industries Inc, 1993.

${ }^{22}$ Brandsma, F. J., Kok, J. C., Dol, H. S., and Elsenaar, A., "Leading Edge Vortex Flow Computations and Comparison with DNW-HST Wind Tunnel Data," Proceedings of the RTO-AVT Symposium on "Advanced Flow Management: Part A Vortex Flows and High Angle of Attack for Military Vehicles" - RTO-MP-069(I) Loen, Norway, NATO RTO, 2001.

${ }^{23}$ Dol, H. S., Kok, J. C., and Oskam, B., "Turbulence Modelling for Leading Edge Vortex Flows," 40th Aerospace Sciences Meeting and Exhibit, AIAA Paper 2002-0843, January 2002.

${ }^{24}$ Spalart, P. R. and Allmaras, S. R., "A One Equation Turbulence Model for Aerodynamic Flows," 30th Aerospace Science Meeting and Exhibit, AIAA Paper 1992-0439, January 1992.

${ }^{25}$ Forsythe, J. R., Hoffmann, K. A., and Dieteker, F. F., "Detached-Eddy Simulation of a Supersonic Axisymmetric Base Flow with an Unstructured Flow Solver," AIAA Paper 2000-2410, June 2000.

${ }^{26}$ Spalart, P. R., "Young Person's Guide to Detached-Eddy Simulation Grids," NASA/CR-2001-211032, NASA Langley Research Centre, July 2001.

${ }^{27}$ Samareh, J., "Gridtool: A Surface Modeling and Grid Generation Tool," Proceedings of the Workshop on Surface Modeling, Grid Generation, and Related Issues in CFD Solution, NASA CP-3291, May 1995.

${ }^{28}$ Pirzadeh, S., "Progress Toward a User-Oriented Unstructured Viscous Grid Generator," AIAA Paper 96-0031, January 1996.

${ }^{29}$ Cummings, R. M., Morton, S. A., and Siegel, S. G., "Computational Simulation and Experimental Measurements for a Delta Wing with Periodic Suction and Blowing," Journal of Aircraft, Vol. 40, No. 5, September-October 2003, pp. $923-931$.

${ }^{30}$ Elsenaar, A. and Hoeijmakers, H. W. M., "An Experimental Study of the Flow over a Sharp-Edged Delta Wing at Subsonic and Transonic Speeds," AGARD Conference Proceedings "Vortex Flow Aerodynamics", AGARD-CP-494, July 1991, pp. 15.1-15.19.

${ }^{31}$ Longo, J. M. A., "Compressible Inviscid Vortex flow of a Sharp Edge Delta Wing," AIAA Journal, Vol. 33, No. 4, April 1995, pp. 680-687.

${ }^{32}$ Morton, S. A., Steenman, M., Cummings, R. M., and Forsythe, J. R., "DES Grid Resolution Issues for Vortical Flows on a Delta Wing and an F-18C," 41st Aerospace Sciences Meeting and Exhibit, AIAA Paper 2003-1103, January 2003.

${ }^{33}$ Morton, S. A., Forsythe, J. R., Squires, K. D., and Wurtzler, K. E., "Assesment of Unstructured Grids for DetachedEddy Simulation of High Reynolds Number Separated Flows," 8th International Conference on Numerical Grid Generation in Computational Field Simulations, June 2002. 